\title{
THEORY OF MAGNETIC SUSPENSIONS IN LIQUID CRYSTALS
}

\author{
F. BROCHARD and P. G. de GENNES \\ Physique des Solides (*), Faculté des Sciences, 91-Orsay \\ (Reçu le 20 mars 1970, révisé le 15 avril 1970)
}

\begin{abstract}
Résumé. - On discute certaines propriétés de particules petites et allongées, en suspension dans un cristal liquide, à basse concentration (fraction en volume $f \lesssim 10^{-3}$ ). On montre que dans la plupart des cas, la direction des particules ne peut pratiquement pas s'écarter de l'axe nématique local $\mathbf{n}(\mathbf{r})$. Si l'on parvenait à préparer de telles suspensions avec des grains magnétiques, on pourrait réaliser des suspensions (( ferronématiques ) ou ( ferrocholestériques ) dans lesquelles, localement, tous les grains sont aimantés dans une même direction (parallèle à n) et aussi des matériaux " compensés ) sans aimantation spontanée. De telles suspensions devraient montrer des distorsions et des changements de phase remarquables en présence de faibles champs extérieurs $H$. On attend aussi des phénomènes de ségrégation : les grains tendent à se déplacer vers les régions du spécimen où $\mathbf{n}$ est parallèle à $\mathbf{H}$. Des effets analogues, sous champ électrique, pourraient en principe être obtenus avec des suspensions colloïdales de macromolécules polaires en bâtonnets, par exemple avec des hélices de polypeptides.
\end{abstract}

\begin{abstract}
The present paper discusses some properties of small, elongated particles suspended in a nematic or cholesteric phase, with low concentrations (typical volume fraction occupied by the particle $f \approx 10^{-3}$ ). We find that in most cases the particle axis should be locked and parallel to the local nematic axis $\mathbf{n}(\mathbf{r})$. If such colloidal suspensions could be made successfully with magnetic grains, it should be possible to prepare ( ferronematics ) or ( ferrocholesterics ), where locally all particles are magnetized in one direction (colinear to $\mathbf{n}$ ), and also (" compensated ) materials with no spontaneous magnetization. These three types of samples should all show remarkable distorsions and phase changes in low external fields $H$. Similar effects are expected under electric fields with colloidal suspensions of polar rod molecules such as helical polypeptides.
\end{abstract}

I. Introduction. - To observe magnetic effects in liquid crystals, we usually require rather large fields $H\left(\sim 10^{4} \mathrm{Oe}\right)$ because the anisotropic part of the diamagnetic susceptibility $\chi_{\mathrm{a}}$ is small $\left(\sim 10^{-7}\right)[1]$. We could try and increase $\chi_{\mathrm{a}}$ by introducing paramagnetic ions with an anisotropic $g$ factor. For strong spin orbit coupling, $g_{/ /}-g_{\perp}$ may be of the order of 0.3. Assuming complete alignment of the magnetic solute, the anisotropic part of the paramagnetic susceptibility is given by

$$
\chi_{/ /}-\chi_{\perp}=\frac{n \mu_{B}^{2} S(S+1)}{3 k_{B} T}\left(g_{/ /}^{2}-g_{\perp}^{2}\right) .
$$

The number $n$ of paramagnetic ions per $\mathrm{cm}^{3}$ cannot be very large, if we want to maintain the mesomorphic properties of the substrate. Typically we could reach $n=10^{20}$, giving $\chi_{/ /}-\chi_{\perp} \sim 10^{-7}$. Thus the paramagnetic susceptibility is not efficient. We may increase the coupling by going to larger magnetic objects, such as ferromagnetic grains. It is not easy to make a colloid with such grains. But, if this turns out to be feasible in a liquid crystal matrix, the resulting

(*) Laboratoire associé au C. N. R. S.

(1) This effect was pointed out to us by J. P. Burger. materials should have remarkable properties, which we discuss in the present paper. How are the grains aligned in the nematic ? We can think of two contributions, one magnetic and one mechanical in origin.

a) EFFeCt OF THE GRAIN MAGNETIC FIELD. - Even for a spherical grain, the distribution of the field around the grain is anisotropic (Fig. 1). Therefore, there will be a preferential direction for the magnetic moment of the grain in a nematic phase $\left({ }^{1}\right)$. But this effect is weak for small grains. Because of the rapid decrease of the field in $1 / r^{3}$, the nematic configuration

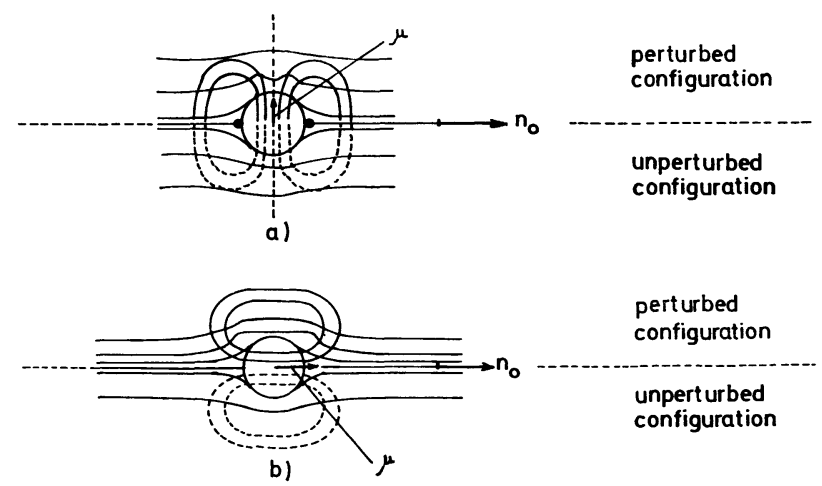

FIG. 1. - Effect of the grain magnetic field on the spatial configuration of the nematic molecules. 
is only perturbed in the neighbourhood of the grain. The free energy, in presence of the magnetic field of the grain, is to first order

$$
F=F_{0}\left(\mathbf{n}_{0}\right)-\frac{1}{2} \chi_{\mathrm{a}} \int\left[\mathbf{n}_{0}(r) . \mathbf{H}(r)_{\lrcorner}{ }^{2} \mathrm{~d} \tau\right.
$$

where $\mathbf{n}_{0}(r)$ is a unit vector describing the unperturbed configuration [2]. $F$ depends upon the angle between the nematic axis $\mathbf{n}_{0}$ and the magnetic moment $\mathbf{u}$. The coupling term $F_{n \mu_{0}^{\prime \prime}}-F_{n \mu_{0}}$, (the difference in energy between the two nematic arrangements shown on Figure $1 a$ and $1 b$ ) is of order $\frac{1}{2} \chi_{\mathrm{a}} \frac{\mu^{2}}{V}$, where $V$ is the grain volume. Typically $\chi_{\mathrm{a}} \sim 10^{-6}, V=10^{-16} \mathrm{~cm}^{3}$, $\mu=10^{-13}$ uem and $\chi_{\mathrm{a}} \frac{\mu^{2}}{V}=10^{-4} \mathrm{eV} \ll k_{B} T$.

b) Mechanical coupling, due to the shape of the grains. We shall see in section II that for an elongated grain with length $\mathrm{L}$ and diameter $d(L / d \sim 10)$, the distortion energy of a nematic liquid crystal is usually minimal if the long axis of the grain coincides with the optical axis. To put the grain in a perpendicular direction requires an energy of the order of $K L$, where $K$ is an average of the Frank elastic constants [2]. $K L$ is of order of $\frac{U L}{a}$, where $U$ is a typical molecular interaction and $a$ is a molecular diameter. $L / a \sim 1000$, thus $E_{\mathrm{mec}} \gg k_{B} T$. Therefore the coupling is mainly of mechanical origin and is very strong in the case of elongated particles.

In section II of the present paper, we analyse first the long range distortions induced in a nematic matrix by a single particle. Then we move on to the case of a dilute suspension, and find the minimum concentration $c_{\min }$ above which the average nematic orientation follows the orientation of the grains. This is roughly $c_{\min } \sim 1 / L D^{2}$ where $D$ is the sample dimension. The corresponding filling factor $f_{\min }=c_{\min } V$ is thus of order $[d / D]^{2}$ where $d$ is the grain diameter : thus $f_{\min }$ is very small and in most experimental situations $f \| f_{\min }$, i. e. the molecular orientation and the grain orientation are along the same direction. A different and more difficult problem occurs at higher concentrations when the particles are magnetic; there is in fact a critical size above which different grains will tend to cluster because of the magnetic interaction. Let us ascertain what is the condition for weak clustering.

Consider rodlike grains $(L / d \sim 10)$. They will not make chains if

$$
f \mathrm{e}^{\Delta E / k_{B} T} \ll 1
$$

where $E$ is the decrease in the demagnetizing field energy when two grains stick together. To estimate $\Delta E$ let us replace the individual grains by ellipsoids of axial ratio $L / d$, and the two-grain system by an ellipsoid of axial ratio $2 L / d$. This gives :

$$
\Delta E=\left[N\left(\frac{L}{d}\right)-N\left(\frac{2 L}{d}\right)\right] M_{s}^{2} V
$$

where $M_{s}$ is the magnetization inside the grains, and $N$ a demagnetization coefficient [3], $(N(10)=0.24$ and $N(20)=0.08)$. Because of the exponential dependence of the left hand side in (I.1) there are in fact two sharply defined regimes:

(1) Small grains : the inequality (I.1) is satisfied for all feasible values of $f$ (i. e. up to $\mathrm{f} \cong 10^{-2}$ ).

(2) Large grains : the inequality (I.1) cannot be satisfied at any physical value of $f$.

Typically, with $M_{s}=10^{3}$ and $L / d=10$ the threshold between (1) and (2) occurs at $L \cong 2000 \AA$. In the present paper we restrict our attention to lengths $L$ below threshold.

In section III we discuss the response of such magnetic suspensions to an external magnetic field $H$; there are two types of effects :

a) Distortions of the nematic (or cholesteric) pattern. These distortions should occur in very low fields : the average magnetization of the suspension, instead of being $\chi_{\mathrm{a}} H\left(\sim 10^{-4}\right.$ to $\left.10^{-3}\right)$ is now $\bar{M}=M_{s} f \cong 0.1$ to 1 . gauss; the gain in coupling is thus of order $10^{3}$.

b) Grain segregation : we have seen that the grains have their moment locked along the local molecular axis $\mathbf{n}(\mathbf{r})$. Thus they tend to move to regions where $\mathbf{n}(\mathbf{r})$ is parallel to the field. These effects should give rise to "depletion layers" of typical thickness 1 to 10 microns, which could be observed optically on thin samples.

II. Floating objects in a nematic phase. II. 1 LONG RANGE DISTORTIONS INDUCED BY A SINGLE OBJECT. - We consider a solid grain, of arbitrary shape, floating in a nematic liquid $\left({ }^{2}\right)$. The surface of the grain may be bare, or it may be coated by a suitable detergent increasing the grain solubility. We assume that the nematic molecules near the grain surface have a well defined orientation: specific examples will be discussed in section II.2. For the moment, we shall not investigate the complicated distortions of the nematic alignment which result in the vicinity of the grain, but only the long range part of these distortions : this turns out to be relatively simple, and also to contain all the physical information which is useful in practice. We restrict our attention to static properties (which are relevant for free energy calculations): the grain under consideration is fixed in position (we put the origin of coordinates at the center of gravity of the grain) and in orientation.

As is usual with nematic systems, we find a consi-

(2) To avoid sedimentation, we require $V\left(\rho_{g}-\rho_{n}\right) g D<k_{B} T$ where $\rho_{g}$ and $\rho_{n}$ are densities of the nematic and of the grain, $g=981 \mathrm{~cm} . \mathrm{s}^{-2}$ and $D$ is the sample dimension. 
derable simplification if the three elastic constants of Frank [2] are taken to be equal

$$
K_{11}=K_{22}=K_{33}=K
$$

in which case the distortion free energy of the nematic takes the simple form

$$
F=\frac{1}{2} K \int \mathrm{d} \tau|\nabla \mathbf{n}|^{2}
$$

where $\mathbf{n}$ is the «director», i. e., the unit vector labelling the preferred axis.

II.1.a Definition of a «distortion amplitude». Far from the grain $(r \gg L) \mathbf{n}$ becomes parallel to the nematic axis $\mathbf{n}_{0}$ (We take the $z$ axis along $\mathbf{n}_{0}$ ). More precisely we may put $\mathbf{n}(\mathbf{r})=\mathbf{n}_{0}+\delta \mathbf{n}(\mathbf{r})$ where

$$
\delta \mathbf{n}\left(n_{x}, n_{y}, 0\right)
$$

is small. Eq. (II.1) leads to a free energy density

$$
\frac{K}{2}\left[\left(\nabla n_{x}\right)^{2}+\left(\nabla n_{y}\right)^{2}\right]
$$

and to the equilibrium equations

$$
\nabla^{2} n_{x}=\nabla^{2} n_{y}=0 .
$$

It is convenient to put

$$
\delta \mathbf{n}(\mathbf{r})=\boldsymbol{\omega}(\mathbf{r}) \wedge \mathbf{n}_{0}
$$

where $\nabla^{2} \omega_{x}=\nabla^{2} \omega_{y}=0$. Since $\omega_{z}$ is arbitrary we may also impose $\nabla^{2} \omega_{z}=0$. Then the most general form of $\omega$ at large $r$ is

$$
\omega(\mathbf{r})=\alpha \frac{1}{r}+\beta: \nabla\left(\frac{1}{r}\right)+\cdots
$$

where $\boldsymbol{\alpha}$ and $\boldsymbol{\beta}$ are respectively a vector and a dyadic, independent of $\mathbf{r}$. We shall mainly focus our attention on the leading term $\alpha / r$. We note first that, in a nematic, the states $\mathbf{n}$ and $(-\mathbf{n})$ are identical. For this reason $\boldsymbol{\omega}$ and $\alpha$ must be even functions of the unperturbed director $\mathbf{n}_{0}$.

We further restrict our attention to grains having an axis of cylindrical symmetry (labelled by a unit vector u). The grain shape may be a cigar (Fig. 2a), a pancake (Fig. 2b), a tapered cylinder (Fig. $2 c$ ) or a helical rod (e. g. a rigid polypeptide) with a definite sign of rotation (Fig. 2d). The vector $\alpha$ depends on both $\mathbf{u}$ and $\mathbf{n}_{0}$. We shall prove first that $\alpha$ is an even function of $\mathbf{u}$. The argument is indicated on figure 3 , where we keep only the term $\alpha 1 / r$ in eq. (II.3) : $\boldsymbol{\omega}$ is then an even function of $\mathbf{r}$. Consider a point (1)

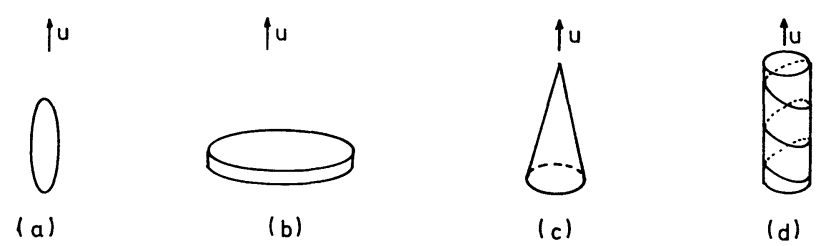

FIG. 2. - Various type of uniaxial grains.
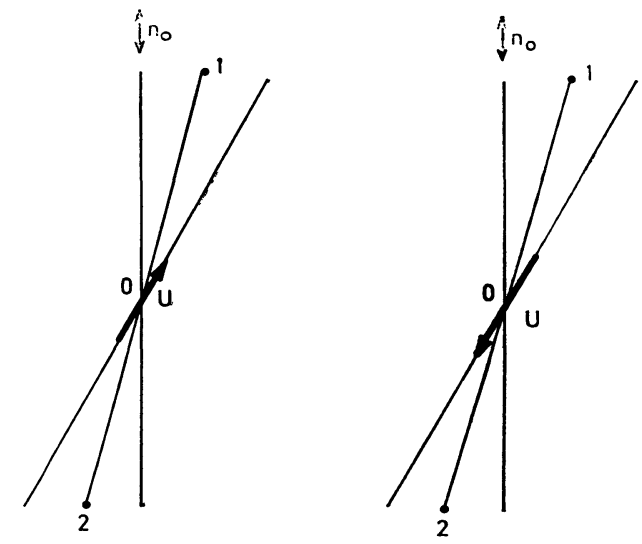

FIg. 3. - Comparison of the distortions corresponding to a grain and the reversed grain.

in the (u, $\left.\mathbf{n}_{0}\right)$ plane and its symmetric (2) with respect to the origin. $\omega_{u}(1) \equiv \omega_{u}(2)$. Now if we rotate both the grain and the nematic by $180^{\circ}$ around an axis $\mathrm{Oy}$ normal to the $\left(\mathbf{u}, \mathbf{n}_{0}\right)$ plane, we see that $\boldsymbol{\omega}_{-u}(2)=\boldsymbol{\omega}_{u}(1)$. Thus $\boldsymbol{\omega}_{u}(2)=\boldsymbol{\omega}_{-u}(2)$ and $\boldsymbol{\omega}_{u}=\boldsymbol{\omega}_{-u}$, Q. E. D.

The most general vector constructed from $\mathbf{u}$ and $\mathbf{n}_{0}$, and even in each of them, is of the form :

$$
\boldsymbol{\alpha}=l(\cos \theta) \mathbf{n}_{\mathbf{0}} \wedge \mathbf{u}
$$

where $\theta$ is the angle $\left(\mathbf{n}_{0}, \mathbf{u}\right)$ and $l(x)$ is an $o d d$ function of $x$. Inserting (II.4) into (II.3) and (II.2) we find for the long range distortions

$$
\delta \mathbf{n}(\mathbf{r})=\frac{l(\cos \theta)}{r} \mathbf{u}_{\perp}
$$

where $\mathbf{u}_{\perp}=\mathbf{u}-\cos \theta \mathbf{n}_{0}$ is the component of $\mathbf{u}$ in the $x y$ plane. Note that the coefficient of $1 / r$ vanishes when $\mathbf{u}$ is parallel to $\mathbf{n}_{0}$ and also when $\mathbf{u}$ is normal to $\mathbf{n}_{0}$ (since $\left.l(0)=0\right)$. The function $l(\cos \theta)$ has the dimensions of a length, and we call it the distortion amplitude. For many small motion problems the quantity of main interest is the value $l(\cos \theta=1)=l_{1}$ (which may be positive or negative). We shall see later that for a long rod $l_{1}$ is usually positive and comparable to the length of the rod.

II.1.b Connection between distortion amplitude and distortion energy. - Consider a surface $\Sigma$ surrounding the grain, but very distant from it. When we impose a fixed value of $\theta$ the grain applies a torque $\Gamma$ on the nematic inside $\Sigma$. Nematics transmit torques: the molecules inside $\Sigma$ exert on the molecules outside $\Sigma$ the same torque $\Gamma$. It is possible to compute this torque, since in the vicinity of $\Sigma$ the distortion has a known form (eq. II.5), and thus to relate $\Gamma$ and $l(\cos \theta)$.

The calculation proceeds as follows: we keep the grain fixed in orientation (u fixed) and we rotate the general nematic direction $\mathbf{n}_{\mathbf{0}}$ by an angle $\delta \theta$ (around an axis normal to $\mathbf{u}$ and $\mathbf{n}_{0}$ : see Fig. 4). The change in 


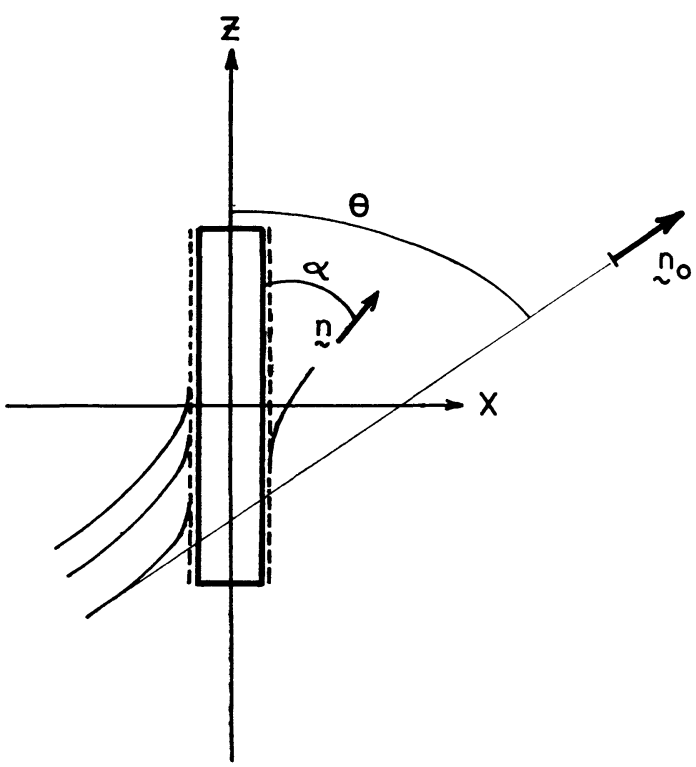

FIG. 4. - Distortion in a nematic induced by a rod $: \mathbf{n}$ is parallel to $Z$ at the grain surface.

distortion energy for the nematic inside $\Sigma$ is to first order

$$
\delta F=\int \mathrm{d} \tau K\left(\partial_{i} n_{j}\right)\left(\partial_{i} \delta n_{j}\right)
$$

where $(i)=x, y, z, \partial_{i} \equiv \partial / \partial_{x_{i}}$ and summations are taken over repeated indices. Integrating by parts we get a volume contribution, which vanishes automatically from the equilibrium equations, and a surface contribution; the integral on the grain surface vanishes and we are left with an integral on $\Sigma$

$$
\delta F=K \int_{(\Sigma)} \mathrm{d} \sigma_{i} \delta n_{j} \partial_{i} n_{j} .
$$

Since $\Sigma$ is a distant surface, we may replace $\delta \mathbf{n}$ by $\delta \mathbf{n}_{0}$ in eq. (II.7) and insert eq. (II.5) to compute $\partial_{i} n_{j}$. The result is

$$
\delta F=K l(\cos \theta) \mathbf{u}_{\perp} . \delta \mathbf{n}_{0} \int \mathrm{d} \sigma_{i} \partial_{i}\left(\frac{1}{r}\right) .
$$

The integral is equal to $-4 \pi$, and the change in distortion energy reduces to

$$
\delta F=4 \pi K l(\cos \theta) \sin \theta \delta \theta .
$$

In vector notation, we may say that the torque $\Gamma$ applied by the grain to the nematic is

$$
\boldsymbol{\Gamma}=4 \pi K l(\cos \theta) \mathbf{n}_{0} \wedge \mathbf{u} .
$$

Thus, to know $f(\cos \theta)$ is equivalent to knowing the $\theta$ dependent part of the distortion energy. For instance, if $l(\cos \theta)$ is positive for $\cos \theta>0$, the distortion energy is a minimum at $\theta=0$ and a maximum at $\theta=\pi / 2$.

II. 2 APPROXIMATE CALCUlation OF THE Distortion AMPLITUDE AND ENERGY. - We consider rodlike parti- cles $(L / d \sim 10)$ imposing a certain boundary condition on the director $\mathbf{n}$ at the grain surface. We shall discuss the following simple cases of boundary conditions :

a) The molecules want to be tangential to the surface and along one preferred direction in this surface. We shall consider the cases where this direction is parallel or perpendicular to the grain axis.

b) Again the molecules are tangential, but there is no preferred direction in the plane of contact; the boundary conditions are degenerate. The elastic equations in the bulk have then more than one solution, and the correct one must be obtained by minimizing $F_{\text {elas }}$ with respect to the molecular orientations inside the plane of contact.

c) The molecules must be perpendicular to the grain surface.

In all three cases $(a)(b)(c)$ we neglect end effects whenever possible. The general argument for this is as follows : the lateral surface of the cylindrical grain is $\sim \mathrm{Ld}$ and the distortion energy (II.1) due to the regions around it is of order

$$
L d K\left(\frac{1}{d}\right)^{2} d \cong K L
$$

since $|\nabla n| \sim 1 / d$ in a thickness $\sim d$ near the surface. On the other hand the ends of the cylinder have a surface $d^{2}$ and contribute an energy

$$
d^{2} K\left(\frac{1}{d}\right)^{2} d \cong K d .
$$

Thus for $d \ll L$ end effects are indeed small.

II.2.a Molecules at the grain surface parallel to the grain axis. - We use coordinates $X, Y, Z$ with $Z$ parallel to the grain axis (Fig. 4) and try a variational solution of the form

$$
\left.\begin{array}{l}
n_{X}=\sin \alpha(\mathbf{r}) \\
n_{Y}=0 \\
n_{Z}=\cos \alpha(\mathbf{r}) .
\end{array}\right\}
$$

This gives :

$$
F=\frac{K}{2} \int(\nabla \alpha)^{2} \mathrm{~d} \tau
$$

and the optimum corresponds to $\nabla^{2} \alpha=0$.

The boundary conditions are $\alpha=0$ on the cylinder and $\alpha=\theta$ at infinity. We may write

$$
\int \frac{(\nabla \alpha)^{2}}{8 \pi} \mathrm{d} \tau=\frac{1}{2} C \theta^{2}
$$

where $C$ is the capacitance of a conducting rod identical in shape to our grain. In practice we shall use for $C$ an approximate value, corresponding not to a rod but to an elongated ellipsoid : 


$$
C \simeq \frac{L}{2 \log \left(\frac{L}{d}\right)}
$$

Using (II.11) and (II.10) we arrive at

$$
F=2 \pi K C \theta^{2} \text {. }
$$

Eq. (II.13) is valid even for large $\theta$; it violates, however, the parity requirement $F(\theta) \equiv F(\pi-\theta)$. In fact, $F(\theta)$ has two branches $F_{1}(\theta)$ and $F_{2}(\theta)$ corresponding to two nematic arrangements (figure 5), and $F_{2}(\theta)=F_{1}(\pi-\theta)$. One may pass from conformation (1) to conformation (2) by introducing a disclination line. We will always stay on the branch of lowest energy if the process is effective.

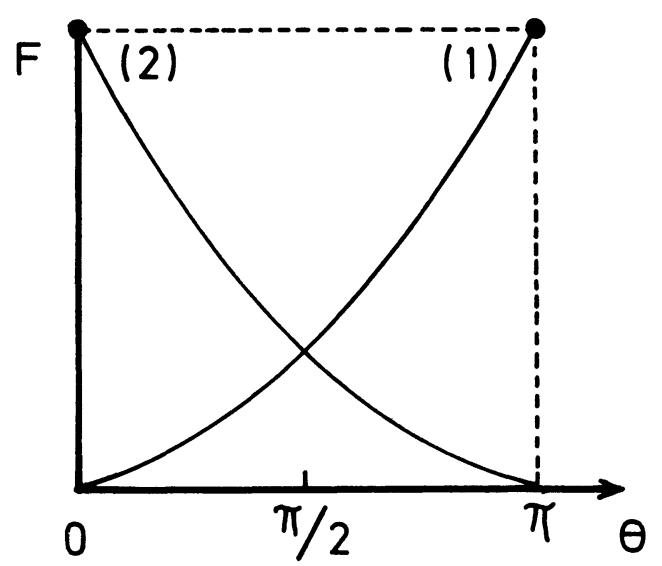

Fig. 5. - Two possible determinations for the energy of the rod as a function of angle.

Note that eq. (II.13) leads to a distortion amplitude $l_{1}=C$ which is essentially proportional to $L$.

II.2.b $\mathrm{n}$ is tangential and perpendicular to $Z$. We restrict ourselves to the case $\theta=\pi / 2$ and $\theta=0$.

For $\theta=\pi / 2$, the parameters are described in figure $6 a$.

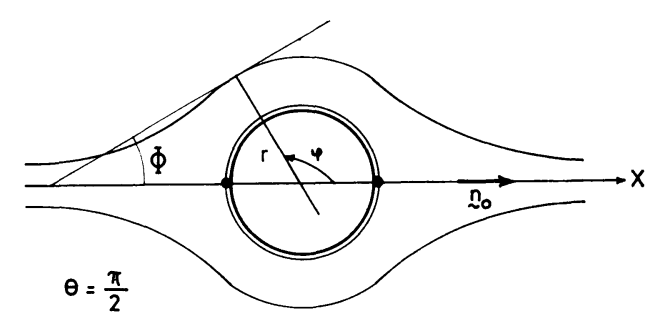

a)

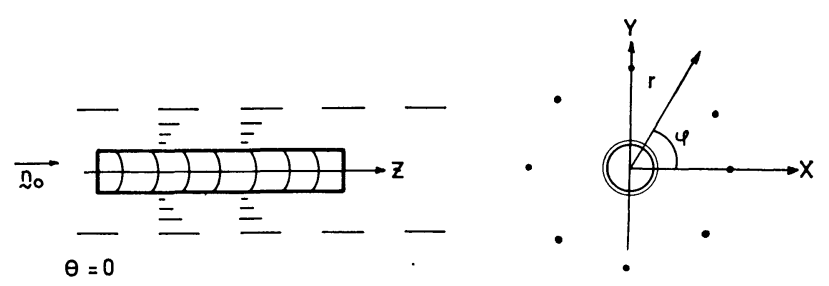

b)

FIG. 6. - Distortion induced by a rod : $\mathbf{n}$ is tangential to the surface and perpendicular to $Z$. n may be written

$$
\left.\begin{array}{l}
n_{X}=\cos \Phi(r, \varphi) \\
n_{Y}=\sin \Phi(r, \varphi) \\
n_{Z}=0
\end{array}\right\}
$$

$F$ is given by $F=\frac{1}{2} \int K(\nabla \Phi)^{2} \mathrm{~d} \tau$

$\delta F=0$ leads to $\nabla^{2} \Phi=0$, i. e.

$$
\frac{\partial^{2} \Phi}{\partial r^{2}}+\frac{1}{r} \frac{\partial \Phi}{\partial r}+\frac{1}{r^{2}} \frac{\partial^{2} \Phi}{\partial \varphi^{2}}=0 .
$$

The solution of this equation is

$$
\Phi=\sum_{n=1}^{\infty} \frac{u_{n} \sin n \varphi+v_{n} \cos n \varphi}{(r / R)^{n}}
$$

where $R=d / 2$ is the grain radius.

$u_{n}$ and $v_{n}$ are determined by boundary conditions. For

$$
\begin{array}{rlrl}
r=R=\frac{d}{2}, & \Phi=\varphi-\frac{\pi}{2} & & 0<\varphi<\pi \\
\Phi & =\varphi-\frac{3 \pi}{2} & \pi<\varphi<2 \pi
\end{array}
$$

$u_{n}$ and $v_{n}$ are the Fourier coefficients of the function of period $\pi$ defined by (II.15) i. e.

$$
u_{2 p}=-\frac{1}{p} \quad v_{n}=u_{2 p+1}=0
$$

$\Phi$ is then given by

$$
\Phi=\sum_{1}^{\infty}-\frac{1}{p} \frac{\sin 2 p \varphi}{(r / R)^{2 p}} .
$$

This may be rewritten in the closed form

$$
\operatorname{tg} \Phi=-\frac{\sin 2 \varphi}{\left(\frac{r}{R}\right)^{2}-\cos 2 \varphi}
$$

This configuration has two disclinations lines attached at the surface at the points

$$
\varphi=0, \quad r=\frac{d}{2} \quad \text { and } \varphi=\pi, \quad r=\frac{d}{2} .
$$

$F$ is readily integrated to give

$$
F=\pi L \log \frac{d}{a}
$$

where $a$ is the radius of the core of the disclination.

For $\theta=0$, the geometry is shown on figure $7 b$. We calculate $F$ by a variational method, choosing as a trial function $\mathbf{n}$ :

$$
\begin{gathered}
n_{r}=0, \quad n_{\varphi}=\frac{a}{\sqrt{(r-a)^{2}+a^{2}}}, \\
n_{z}=\frac{r-a}{\sqrt{(r-a)^{2}+a^{2}}} .
\end{gathered}
$$




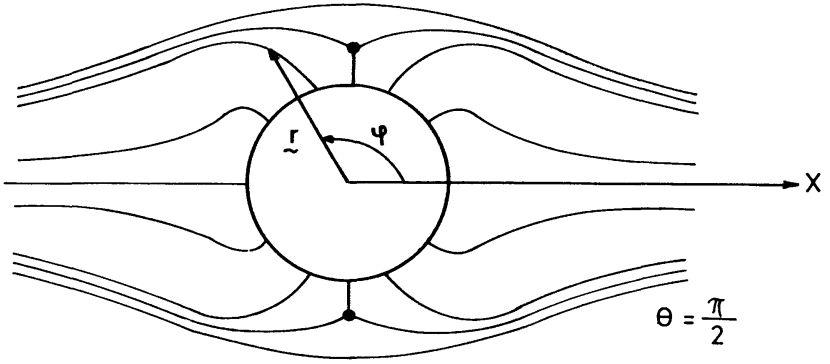

a)

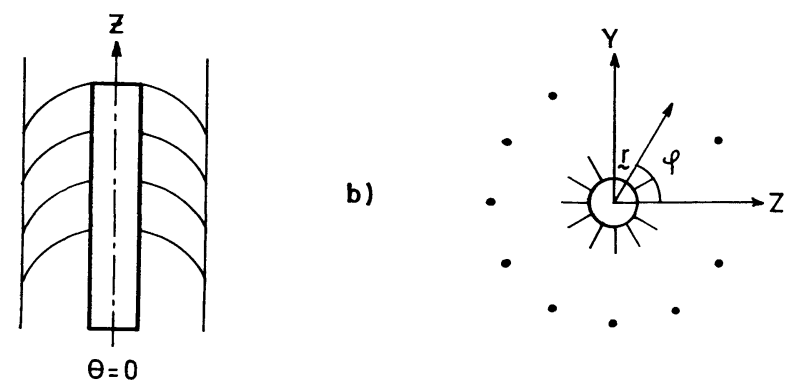

FIG. 7. - Distortion induced by a rod : $\mathbf{n}$ is perpendicular to the grain surface.

$F$ is readily integrated to give

$F_{/ /}=\pi K L \int_{0}^{\infty} \mathrm{d} x \frac{x^{2}+(x+1)^{2}}{(x+1)\left(x^{2}+1\right)^{3}} \simeq \pi K L$.

We note that $F_{/ /}<F_{\perp}$. We find that

$$
F_{\perp}-F_{/ /} \simeq \pi K L \log \frac{d}{\mathrm{e} . \mathrm{a}}
$$

is large and not very sensitive to the diameter of the grain ( $e$ is the base of Neperian logarithms).

II.2.c $\mathbf{n}$ is perpendicular to the grain surface.We restrict ourselves to the cases $\theta=\pi / 2$ and $\theta=0$. $\theta=\pi / 2$ (see Fig. 8a). This case is identical to $1 . b$ except for a $90^{\circ}$ rotation of the molecules. The configuration has two lines attached at the surface at the points

$$
\varphi=\frac{\pi}{2}, \quad r=\frac{d}{2} \quad \text { and } \quad \varphi=\frac{3 \pi}{2}, \quad r=\frac{d}{2} .
$$

The energy $F$ is still given by eq. (II.17). Note that if the disclination line separates slightly from the grain surface, this may release some strain (because of image force effects). However we do not expect this to alter very much $F_{\perp}$.

$\underline{\theta=0}$ (see Fig. $7 b$ ). We calculate $F$ by a variational method, choosing as a trial function $\mathbf{n}$ :

$n_{r}=\frac{a}{\sqrt{(r-a)^{2}}} \overline{\overline{+a^{2}}}, \quad n_{\varphi}=0, \quad n_{z}=\frac{r-a}{\sqrt{(r-a)^{2}+a^{2}}}$.

$F$ is readily integrated to give

$$
F_{/ /}=\pi K L \int_{0}^{\infty} \mathrm{d} x \frac{x^{4}+\left(x-1+x^{2}\right)^{2}}{(x+1)\left(x^{2}+1\right)^{4}} \simeq \frac{\pi K L}{4} .
$$

Again we find that $F_{/ /}<F_{\perp}$ and

$$
F_{\perp}-F_{/ /} \simeq \pi K L \log \frac{d}{a} \text {. }
$$

Conclusions. - If the grain surface aligns the first layer of nematic molecules surrounding it, we find that $F_{/ /}<F_{\perp}$ for an elongated grain and that

$$
F_{\perp}-F_{/ /}=\alpha K L
$$

where $\alpha$ is a numerical coefficient of the order of unity and is not very sensitive to the diameter of the grains.

II. 3 FINITE CONCENTRATIONS OF GRAINS: TRANSITION FROM INDIVIDUAL TO COLLECTIVE BEHAVIOUR. We now consider a dilute suspension of identical grains, with position $\mathbf{r}_{p}$ (distributed at random) and orientation $\mathbf{u}_{p}$, in a nematic of optical axis $\mathbf{n}_{0}$. For simplicity we shall assume that misalignments are small $\left(l_{1}>0, \mathbf{u}_{p \perp} \ll 1\right)$. The distortion $\delta \mathbf{n}(\mathbf{r})$ is then given by the following equation :

$$
\delta \mathbf{n}(\mathbf{r})=\sum_{p} \frac{l_{1}}{\left|\mathbf{r}-\mathbf{r}_{p}\right|}\left[\mathbf{u}_{p \perp}-\delta n\left(\mathbf{r}_{p}\right)\right]
$$

The presence of $\delta n\left(\mathbf{r}_{p}\right)$ in the bracket expresses the fact that grain $(p)$ creates no long range distortion if it is aligned in the local nematic direction $\mathbf{n}_{0}+\delta n\left(r_{p}\right)$. Let us now go to a continuum approximation : if $C$ is the number of grains per $\mathrm{cm}^{3}$ we find, after acting with the operator $\nabla^{2}$ on both sides of equation (II.21) :

$$
\begin{aligned}
\nabla^{2} \delta \mathbf{n} & =-\kappa^{2}\left[\mathbf{u}_{\perp}-\delta \mathbf{n}\right] . \\
\kappa^{2} & =4 \pi C l_{1}>0 .
\end{aligned}
$$

The solution is

$$
\delta \mathbf{n}(\mathbf{r})=C l_{1} \int \mathrm{d} \mathbf{r}^{\prime} \frac{1}{\left|\mathbf{r}-\mathbf{r}^{\prime}\right|} \mathrm{e}^{-\kappa\left|r-r^{\prime}\right|} \mathbf{u}_{\perp}\left(\mathbf{r}^{\prime}\right) .
$$

Thus the effect of each grain is screened out at distances larger than $\kappa^{-1}$. Typically with $C=10^{12}$ and $l_{1}=10^{3} \AA$ we expect $\kappa^{-1} \sim 1 \mu$.

Now let us assume that, in a certain region $R$ of the sample (with linear dimensions $D$ ) we impose a fixed value of $\mathbf{u}_{\perp}$, while out this region we put $u_{\perp}=0$ (Fig. 8). Will the nematic molecule in $R$ follow the grain direction or not ? The answer is provided by eq. (II.23). When $D \gg \kappa^{-1}, \delta \mathbf{n}$ is indeed identical to $\mathbf{u}_{\perp}$

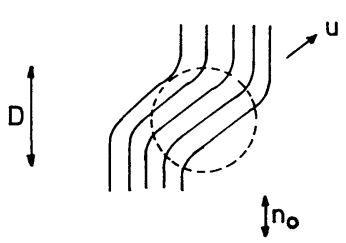

a)

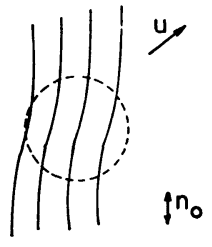

b)
Fig. 8. - Coupling between the grains and the nematic. In a region of size $D$ the grains have an imposed orientation $\mathbf{u}$. Outside of $D$ the grains are parallel to the nematic direction at rest $n_{0}$. Case $(a) \kappa D>1$ : all the inside follows the grains. Case $(b) \kappa D<1$ : the inside is only weakly perturbed. 
in all the region $R$ (except for a thin shell of thickness $\kappa^{-1}$ near the surface) : the grains impose their orientation. In the opposite limit $\left(D \ll \kappa^{-1}\right), \delta \mathrm{n} \ll u_{\perp}$, i. e. the nematic response is small.

II. 4 Right HANDED AND LEFT HANDED GRAINS. The function $l(\cos \theta)$ is the same for a grain and for its mirror image : the terms of order $1 / r$ in the long range distortion are not sensitive to chirality effects. To display an interesting difference between right and left handed grains we must include the terms of order $1 / r^{2}$. These terms will be dominant only when $\alpha=0$. This can be achieved in two ways :

a) With a very small grain, the coupling energy between $\mathbf{u}$ and $\mathbf{n}_{0}$ (of order $4 \pi K l_{1}$ ) may become smaller than $k_{B} T$ : then $\mathbf{u}$ points with equal probability in all directions and the average of $\alpha$ may be shown to vanish, from eq. (II.4).

b) With a helical rod (polypeptide, virus, ...), equilibrium will often correspond to $\mathbf{u}$ parallel to $\mathbf{n}_{0}$, in which case $\alpha=0$.

Let us assume that we are in such a situation : then the only distortion term compatible with rotational invariance, and with the fact that $\boldsymbol{\omega}$ is even in $\mathbf{n}_{0}$, is :

$$
\omega=\beta \nabla\left(\frac{1}{r}\right)
$$

where $\beta$ is a pseudoscalar parameter (with the dimensions of a square length), and thus non vanishing only if the floating object differs from its mirror image. The aspect of the corresponding distortion is shown on figure 9 .

Let us discuss now the case of a dilute solution of such objects in a nematic, all grains having the same chirality. On a macroscopic scale, we know that such
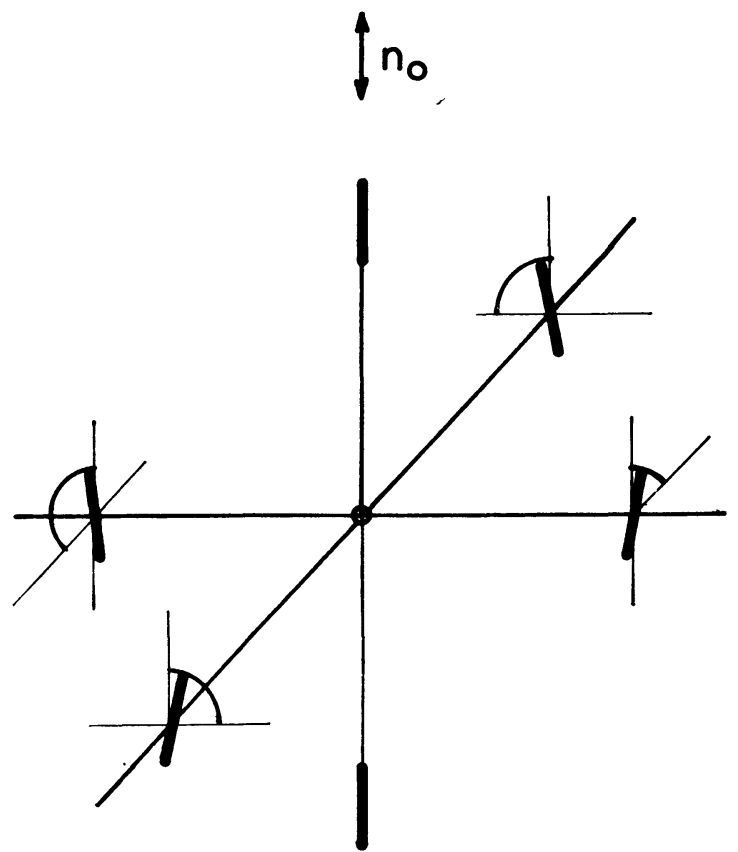

FIG. 9. - Distortion in a nematic induced by a chiral impurity (i. e. an impurity different from its mirror image). a system behaves as a cholesteric. We shall show that there is a simple relation between the pitch $L_{\mathbf{c}}$ of the spiral structure, the concentration $v$ and the parameter $\beta$ defined above : for low $v$ values the total rotation vector at any point $\mathbf{r}$ is obtained by superposition :

$$
\omega(\mathbf{r})=\beta \sum_{p} \nabla \frac{1}{\left|\mathbf{r}-\mathbf{r}_{p}\right|} .
$$

Going to the continuum limit, this leads to

$$
\begin{aligned}
& \operatorname{div} \omega=-4 \pi \beta v \\
& \operatorname{curl} \omega=0 .
\end{aligned}
$$

The form of the solution is

$$
\begin{aligned}
& \omega_{z}=-4 \pi \beta v z+\text { const } \\
& \omega_{x}=\omega_{y}=0
\end{aligned}
$$

where $z$ is an arbitrary axis. This corresponds to a cholesteric structure of half pitch

$$
\pi\left|\frac{\partial z}{\partial \omega_{z}}\right|=\frac{1}{4 \beta v}
$$

inversely proportional to the concentration. This type of law is indeed familiar for dilute solutions [4].

III. Magnetic grains. - III. 1 Properties of A SINGLE (MONODOMAIN) PARTICLE. - III.1. a) Ferromagnetism vs superparamagnetism. - We now give to our particle (of length $L$, diameter $d$, volume $V$ ) a magnetic moment $\mu=M_{s} V$. We assume for simplicity that the moment $\boldsymbol{\mu}$ has two easy directions — parallel and antiparallel to the grain axis, and that it cannot jump from one direction to the other (ferromagnetic grain). If $\Delta E$ is the energy barrier between these two conformations, this requirement implies [5]

$$
\Delta E \subsetneq 10 k_{B} T \text {. }
$$

Possible sources for $\Delta E$ are : $(\alpha)$ an uniaxial magnetocrystalline energy $-(\beta)$ the shape anisotropy

$$
\Delta E=\pi M_{s}^{2} T
$$

$-(\gamma)$ surface effects $\left({ }^{3}\right)$. In cases $(\alpha)$ and $(\beta) \Delta E$ is proportional to the grain volume $V$, and the inequality (III.1) gives a minimum value for $V$. In most cases of interest here, $V$ will be significantly larger than this minimum.

III.1. b) Mechanical clamping. - As shown in section II, the grain usually tends to be parallel to the local nematic axis $\mathbf{n}(\mathbf{r})$. To rotate the grain materially from $+\mathbf{n}$ to $-\mathbf{n}$, we need an energy $\alpha K L$. The grain will be effectually clamped in one of the two positions provided that

$$
t D_{r} \exp \frac{-\alpha K L}{k_{\mathrm{B}} T} \sim 1
$$

(3) We are indebted to the referee of the present paper for pointing out the importance of $(\alpha)$ and $(\gamma)$ in the cases of interest. 
where $t$ is the duration of the experiment, and $D_{r}$ is the rotational diffusion constant of the grain (in an isotropic liquid of viscosity similar to that of the nematic). This condition for mechanical clamping may be rewritten qualitatively as

$$
L \subsetneq \frac{10 k_{\mathrm{B}} T}{K} \sim 50 \AA
$$

and it should be satisfied in most practical situations.

III.1. c) Coercive fields of a single grain. - To rotate $\mathbf{u}$ from, say, $\mathbf{n}$ to $-\mathbf{n}$ in direction, we must apply a strong field $\mathbf{H}$ along $-\mathbf{n}$. Two types of behaviour may be found : if $\mathbf{u}$ rotates without any mechanical motion of the grain the threshold is [5]

$$
H_{\text {mag }}=2 \pi M_{s}
$$

while if the grain rotates materially, the threshold is

$$
H_{\mathrm{mech}}=\frac{K l_{1}}{\mu}
$$

where $l_{1}$ is the characteristic length defined in section II, and is of order $L$. Taking the ratio of both coercive fields we find

$$
\frac{H_{\mathrm{mech}}}{H_{\mathrm{mag}}}=\frac{K l_{1}}{2 \pi M_{s}^{2} V} \cong \frac{K}{M_{s}^{2} d^{2}}=\left(\frac{d_{\mathrm{c}}}{d}\right)^{2} .
$$

The critical diameter $d_{\mathrm{c}}$ is of order 50 to $100 \AA$. For $d>d_{\mathrm{c}}$ the dominant process is mechanical rotation. The various types of behaviour of a single grain as a function of $L$ and $d$ are summarized on Figure 10.

A typical value of $H_{\text {mech }}$ (for $d=200 \AA, M_{s}=10^{3}$ and $l_{1} \sim L$ ) would be 300 œrsteds $\left({ }^{4}\right)$. Thus, with moderate fields in the kiloœrsted range, it should

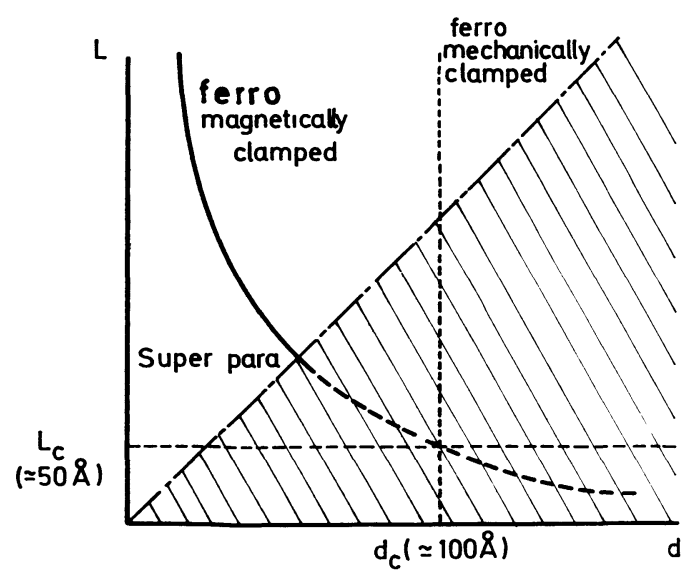

FIG. 10. - Domains of mechanical and magnetical clamping of a single domain magnetic grain in a liquid crystal. $\mathrm{L}$ and $\mathrm{d}$ are respectively the length and diameter of the grains. The only meaningful regions correspond to $L \gg d$.

(4) If the grain (or the surrounding distortions) is large enough to be observed optically, it might be possible to measure the distortion amplitude $f(\theta)$ from a study of $\theta(\mathbf{H})$ for a single grain in a thin nematic slab. be possible to align the grains completely. We shall now proceed to discuss suspensions which have been prepared in such a magnetized state: we shall see in particular that when $H \ll H_{\text {mech }}$, although individual rotations are forbidden, cooperative rotations, involving many grains, may still occur.

III.2 Ferronematics. - III.2 a Free energies and segregation effects. - We now consider a suspension (with low volume fraction of grains $f$ ) and assume that locally all grains, in each small volume element, have parallel moments. As shown above, this common direction is colinear to the director $\mathbf{n}(\mathbf{r})$ and, by a suitable definition of $\mathbf{n}$, we may take it to be of the same sign as $\mathbf{n}$. The average magnetization vector is then

$$
\mathbf{M}=M_{s} f \mathbf{n}
$$

and the free energy density takes the form

$$
\begin{aligned}
& F=\frac{1}{2}\left[K_{11}(\operatorname{div} \mathbf{n})^{2}+K_{22}(\mathbf{n} \cdot \operatorname{curl} \mathbf{n})^{2}+\right. \\
& \left.+K_{33}(\mathbf{n} \wedge \operatorname{curl} \mathbf{n})^{2}\right]-M \mathbf{n} \cdot \mathbf{H}+\frac{f k_{B} T}{V} \log f .
\end{aligned}
$$

The first term is the Frank elastic energy [2] and the last one is the entropy contribution for a dilute suspension of non interacting grains. If we minimize (III.7) first with respect to $\mathbf{n}$, we find that the torque on the nematic molecules contains the usual elastic terms plus a magnetic term $\bar{M} \mathbf{n} \wedge \mathbf{H}$. Minimization with respect to $f$ (keeping the number of grains fixed) leads to :

$$
f=f_{0} \exp \left[\frac{\mu \mathbf{H} \cdot \mathbf{n}}{k_{B}} \frac{T}{T}\right] .
$$

The parameter $f_{0}$ is obtained as usual from the total number of grains present $N$ :

$$
N=\int \frac{f}{\mathrm{~V}} \mathrm{~d} \tau \text {. }
$$

Eq. (III.8) shows that $f$ may vary strongly from point to point, i. e., grain segregation may occur, provided that the number

$$
\rho=\frac{\mu H}{k_{B} T}=\frac{M_{s} V H}{k_{B} T}
$$

be larger than 1 . We call $\rho$ the segregation parameter. Specific examples with $\rho<1$ and with $\rho>1$ will be discussed later. To end the discussion of free energies we note that after minimization the total free energy $\mathcal{F}$ of the sample becomes simply

$$
\mathscr{F}=\mathscr{F}_{\text {el }}+N k_{B} T \log f_{0}+\text { constant }
$$

as can be seen from eqs. (III.8) (III.7). Here $\mathbf{F}_{\mathrm{el}}$ is the contribution of the Frank terms, integrated over the sample volume. We shall now apply these formulas to some selected experimental situations. 
III. 2.b Alignment near a wall. - Consider a ferronematic occupying the half space $z>0$. Assume that on the limiting wall (i. e. the plane $z=0$ ) the molecules must be directed along the $x$ direction. The sample has been prepared with a field $H$ along $x$; later we rotate $H$ and bring it along the $y$ direction. Far in the liquid, the magnetization will also rotate by $90^{\circ}$. But near the wall there must be a transition layer, the thickness and properties of which we want to study (Fig. 11).

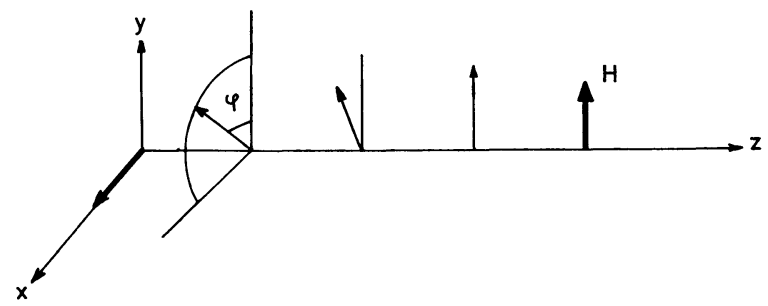

Fig. 11. - Distortion in a ferronematic near an oriented wall.

At all points $r$ the director $\mathbf{n}(r)$ will be in the $(x y)$ plane and we may define it through the angle $\varphi$ between $\mathbf{n}$ and the $y$ axis. For $z=0, \varphi=\pi / 2$; for $z \rightarrow \infty$ $\varphi \rightarrow 0$.

The free energy per unit area of the wall is

$$
\begin{aligned}
F=\int_{0}^{\infty} \mathrm{d} z\left[\frac{1}{2}\right. & K_{22}\left(\frac{\mathrm{d} \varphi}{\mathrm{d} z}\right)^{2}- \\
& \left.\quad-f M_{s} H \cos \varphi+\frac{f k_{B} T}{V} \log f\right]
\end{aligned}
$$

and the equilibrium equation for $\varphi$ has the form

$$
-K_{22} \frac{\mathrm{d}^{2} \varphi}{\mathrm{d} z^{2}}+f(z) M_{s} H \sin \varphi=0 .
$$

It is convenient to define a reduced concentration $\psi=f / \bar{f}$, where $\bar{f}$ is the average of $f$ over the sample volume, and a "coherence length » $\xi_{2}(H)$ by the equation

$$
\xi_{2}^{2}=\frac{K_{22}}{M_{s} \bar{f} H} .
$$

Typically for $H=1$ gauss, $\bar{f}=10^{-3}$, and $M_{s}=10^{3}$, $\xi_{2}=10$ microns. For the present case, eq. (III.8) leads to

$$
\psi=\exp [\rho(\cos \varphi-1)]
$$

and eq. (III.12) has the first integral

$$
\frac{\rho \xi_{2}^{2}}{2}\left[\frac{\mathrm{d} \varphi}{\mathrm{d} z}\right]^{2}+\psi(z)=1 .
$$

The choice of integration constant in eq. (III.15) is such that $\mathrm{d} \varphi / \mathrm{d} z=0$ for $z \rightarrow \infty(\psi \rightarrow 1)$. Integrating (III.15) we arrive at an implicit relation between $\varphi$ and $z$ :

$\frac{z}{\xi_{2}}=\int_{\varphi}^{\pi / 2} \mathrm{~d} \theta\left\{\frac{2}{\rho}[1-\exp (\rho \cos \theta-\rho)]\right\}^{-1 / 2}$.
We shall discuss first the simple limiting case $\rho \ll 1$ (no segregation). Then eq. (III.16) simplifies considerably, and the final result is :

$$
\operatorname{tg} \varphi / 4=\operatorname{tg} \pi / 8 \mathrm{e}^{-z / \xi_{2}}
$$

The decrease of $\varphi$ with $z$ is nearly exponential, and the thickness of the transition layer is equal to the coherence length $\xi_{2}$.

In the opposite limit $\rho>1$ segregation effects become important; the general aspect of $\varphi(z)$ and of $\psi(z)$ is shown on figure 12. At very large distances $z$ from the wall, $\varphi(z)$ again decreases like

$$
\exp (-z / \xi) \text {. }
$$

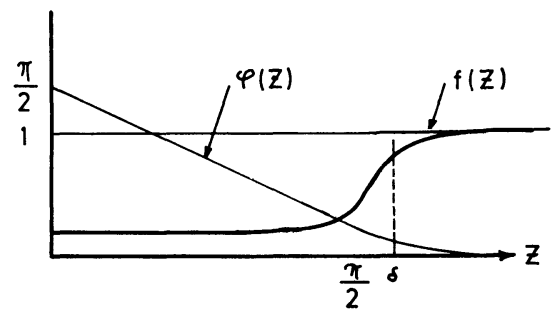

FIG. 12. - Structure of the depletion layer $(\rho>1)$ for the geometry of figure 12 .

However, this law does not hold down to $z \sim \xi_{2}$ and the transition region is larger than $\xi_{2}$. Its thickness may be estimated from the initial slope :

$$
\begin{aligned}
\left.\frac{\mathrm{d} z}{\mathrm{~d} \varphi}\right|_{z=0}=\xi\left[\frac{\rho}{2(1-\psi(z=0))}\right]^{1 / 2}= \\
=\xi\left[\frac{\rho}{2\left(1-\mathrm{e}^{-\rho}\right)}\right]^{1 / 2}
\end{aligned}
$$

where we have used eq. (III. 15, 14). In the high field limit $(\rho>1)$ this characteristic length becomes

$$
\delta=\xi \frac{\sqrt{\rho}}{2}=\left[\frac{K_{22}}{2 c k_{B} T}\right]^{1 / 2}
$$

where $c=\bar{f} / V$ is the average grain concentration in the bulk. The thickness $\delta$ is independent of the magnitude of $H$, and is typically of order 10 microns. In a depth $\delta$ near the wall, the grain concentration is strongly reduced $\left(f \mid \bar{f} \simeq \mathrm{e}^{-\rho}\right)$ : thus a homogeneous field of suitable orientation will create a depletion layer near the wall. The work required to expel the grains from the layer is (per unit wall area) of order $c k_{B} T \delta$ ( $c k_{B} T \delta$ being the osmotic pressure), while the torsion energy is of order $K_{22} \delta / \delta^{2}$. Minimizing the sum, one recovers eq. (III.19), except for numerical coefficients. It would be of great interest to try anci observe these depletion layers by optical means.

III.2.c Ferronematic slab in reversed fields : instability threshold. - We consider a thin slab (thickness $D \sim 10$ microns) of our nematic suspension, enclosed between two glass walls polished according to the method of Chatelain [6]. Both walls are polished along the same direction $(x)$ : near them, the director $\mathbf{n}$ 
must be colinear to $x$. We call $z$ the direction normal to the walls (Fig. 13). We assume that the sample has been prepared with a large field applied along the negative $x$ direction (giving full saturation and a uniform grain concentration). Now we reverse the field, and go to small positive values of $H$. The initial configuration is metastable, and becomes completely unstable at a certain threshold field $H$, which we shall now derive.

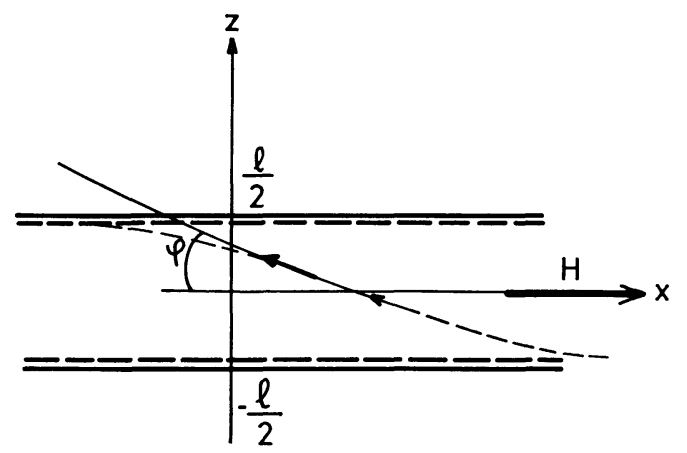

FIG. 13. - Ferronematic slab in reversed field.

Consider a small deviation from the original arrangement, where the director $\mathbf{n}$ has the following structure :

$$
\left.\begin{array}{l}
n_{x} \simeq 1 \\
n_{y}(z) \ll 1 \\
n_{z}(z) \ll 1
\end{array}\right\}
$$

Inserting this form into eq. (III.7) one gets, for the free energy per unit wall area

$$
\begin{aligned}
f= & \int_{0}^{D} \mathrm{~d} z \frac{1}{2}\left[K_{11}\left(\frac{\mathrm{d} n_{z}}{\mathrm{~d} z}\right)^{2}+\right. \\
& \left.+K_{22}\left(\frac{\mathrm{d} n_{y}}{\mathrm{~d} z}\right)^{2}-\bar{M} H\left(n_{x}^{2}+n_{y}^{2}\right)\right]+ \text { constant }
\end{aligned}
$$

(Note that this result is the same with or without segregation effects.) We expand $n_{y}$ and $n_{z}$ in Fourier series matching the boundary conditions at both walls :

$$
\begin{aligned}
& n_{y}=\sum_{1}^{\infty} v_{n} \sin \left(\frac{n \pi z}{D}\right) \\
& n_{z}=\sum_{1}^{\infty} \mu_{n} \sin \left(\frac{n \pi z}{D}\right) .
\end{aligned}
$$

In terms of the $\mu^{\prime} \mathrm{s}$ and $v^{\prime} \mathrm{s}$ the free energy becomes

$$
\begin{aligned}
f=\frac{D}{4} \sum_{1}^{\infty}\left\{v_{n}^{2}\left[K_{11}\left(\frac{n \pi}{D}\right)^{2}-\bar{M} H\right]+\right. \\
\left.+\mu_{n}^{2}\left[K_{22}\left(\frac{n \pi}{D}\right)^{2}-\bar{M} H\right]\right\} .
\end{aligned}
$$

Instability occurs when one the terms becomes negative ; this happens first for $n=1$. There are two possible threshold fields

$$
\left.\begin{array}{l}
H_{s}=\frac{K_{11} \pi^{2}}{\bar{M} D^{2}} \\
H_{t}=\frac{K_{22} \pi^{2}}{\bar{M} D^{2}}
\end{array}\right\} \text { (twist instability). }
$$

The physical threshold $H_{1}$ is the smallest of the two. Taking $\bar{M}=f M_{s}=1$ gauss, $D=10$ microns and $K \sim 10^{-6}$ we have $H_{1} \sim 10$ gauss. Thus, even for rather small samples, a coupled rotation of many grains is able to relax the magnetic moment much more efficiently than individual rotations: let us compare $H_{1}$ to the coercive field for one grain $H_{\text {mech }}$ (eq. III.3). Neglecting all numerical coefficients we find

$$
\frac{H_{\mathrm{c}}}{H_{\mathrm{mech}}} \simeq \frac{d^{2}}{\bar{f} D^{2}} \simeq \frac{1}{(\kappa D)^{2}}
$$

where $\kappa^{-1}$ is the "screening" length introduced in eq. (II.22). Eq. (III.23) shows that collective behavior is observable provided that $\kappa D>1$, in exact agreement with our conclusions of section II.

III.2.d Behavior above threshold. - This part of the problem is more delicate, for various physical reasons. Here we shall restrict our attention to one typical case, defined by the following assumptions :

- Segregation effects are negligible $(\rho<1)$.

- All elastic constants are equal : in this case it is plausible to assume that there is no twist, $i$. e. that the director $\mathbf{n}$ is always in the $(x, z)$ plane. We shall specify its orientation through the angle $\varphi(z)$ between $\mathbf{n}$ and $\mathbf{H}$.

- No disclination line is allowed (we come back to this point in the next paragraph).

With these assumptions the equilibrium equation retains the form (III.12) (with a constant $f$ ). Eq. (III.15) is replaced by

$$
-\frac{\xi^{2}}{4}\left(\frac{d \varphi}{d z}\right)^{2}+\sin ^{2} \frac{\varphi}{2}=\sin ^{2} \frac{\varphi_{m}}{2}
$$

where $\varphi_{\mathrm{m}}$ is the deflexion angle at the middle of the slab. The solution $\varphi(z)$ may be expressed in terms of elliptic functions. The boundary condition

$$
\varphi(0)=\varphi(D)=\pi
$$

leads to an implicit equation for $\varphi_{\mathrm{m}}$

$$
\begin{aligned}
D=2 \xi \int_{0}^{\pi / 2} & \frac{\mathrm{d} u}{\sqrt{1-\sin ^{2} \frac{\pi-\varphi_{\mathrm{m}}}{2} \sin ^{2} u}}= \\
& =2 \xi F\left(\frac{\pi}{2}, \sin \frac{\pi-\varphi_{\mathrm{m}}}{2}\right) .
\end{aligned}
$$

Our definition of the elliptic integrals such as $F$ follows ref. [7].

Eq. (III.25) has a solution only for $H \geqslant H_{1}$. 
The spatial configuration of the molecules for various $H$ values above $H_{1}$, are shown on figure 14 . As soon as $H \gg H_{1}$ the bulk of the nematic is magnetized parallel to $\mathbf{H}$, and there are only two thin regions of thickness $\xi$ near the walls where the magnetization is mainly in the wrong direction. It is also of interest to compute the average magnetization

$$
\begin{gathered}
M_{\mathrm{av}}=\frac{\bar{M}}{D} \int_{0}^{D} \mathrm{~d} z \cos \varphi \\
\frac{M_{\mathrm{av}}}{\bar{M}}=1-\frac{2 E\left(\frac{\pi}{2}, \sin \frac{\pi-\varphi_{\mathrm{m}}}{2}\right)}{F\left(\frac{\pi}{2}, \sin \frac{\pi-\varphi_{\mathrm{m}}}{2}\right)} .
\end{gathered}
$$

A plot of $M_{\mathrm{av}} / \bar{M}$ versus $H$ for this ideal case is shown on figure 15.
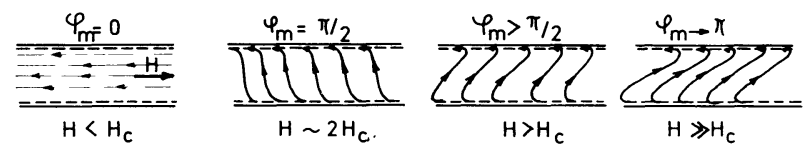

FIG. 14. - Spatial configuration of the molecules for various $H$ values.

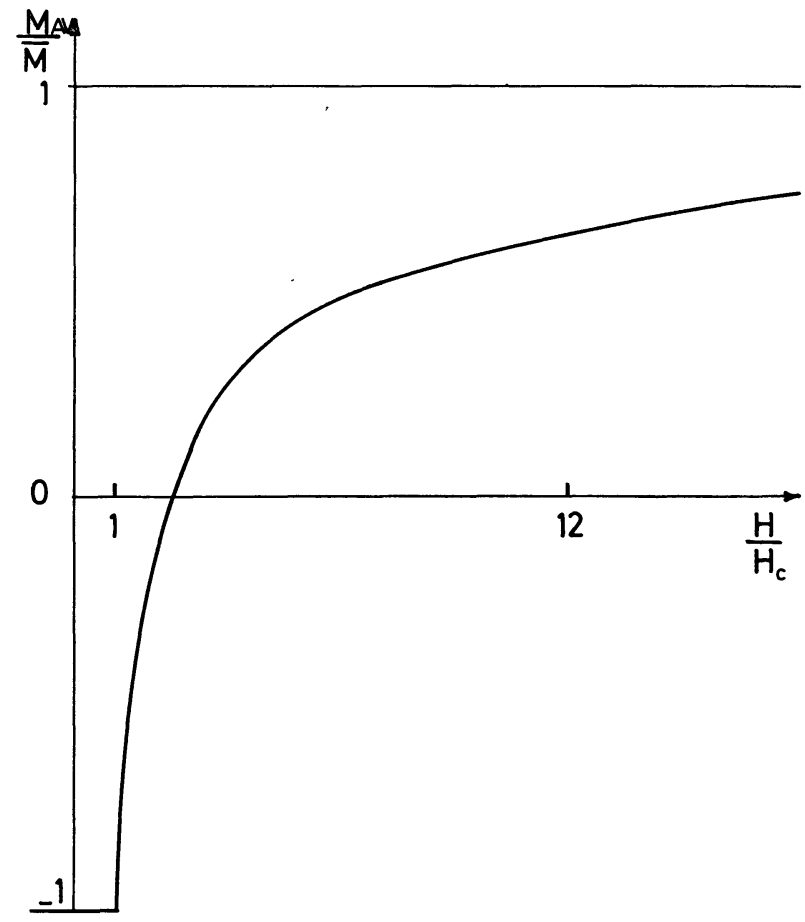

FIG. 15. - Magnetization curve of a ferronematic slab.

III.2.e Possible effects of disclinations. - The conformations which we have described above, with a magnetized nematic in a reversed field, are metastable. They might be disrupted by the nucleation and motion of disclination lines [8]. We shall discuss here some specific features of these lines occurring when magnetic grains are present.

As pointed out earlier, in most cases the average magnetization $\mathbf{M}(\mathbf{r})$ is parallel to the local nematic axis (i. e. parallel or antiparallel to the director $\mathbf{n}$ ). Consider now the disclination loop $L_{0}$ shown on figure 16 . We assume that $L_{0}$ is of rank 1 , i. e., if we follow a circuit such as $C$, starting from a director $\mathbf{n}$ at point 1 we obtain a director $-\mathbf{n}$ at the same point after one turn.

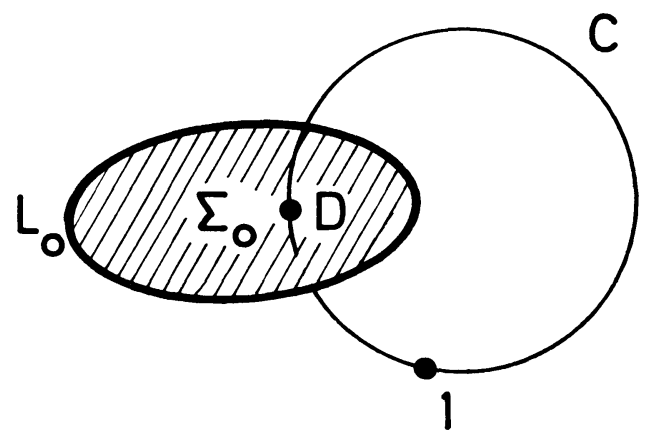

FIG. 16. - Disclination line $L_{0}$ and mismatch surface $\Sigma_{0}$ in a ferronematic.

This shows that $\mathbf{M}$ cannot be constantly parallel to and of the same sign as $\mathbf{n}:$ there must be at least one discontinuous point $D$ on $C$. The locus of these points is a surface of discontinuity $\Sigma_{0}$, bounded by $L_{0}$. When we cross $\Sigma_{0}$, we find an abrupt $180^{\circ}$ rotation of the vector M. An example is shown on figure $17\left({ }^{5}\right)$.

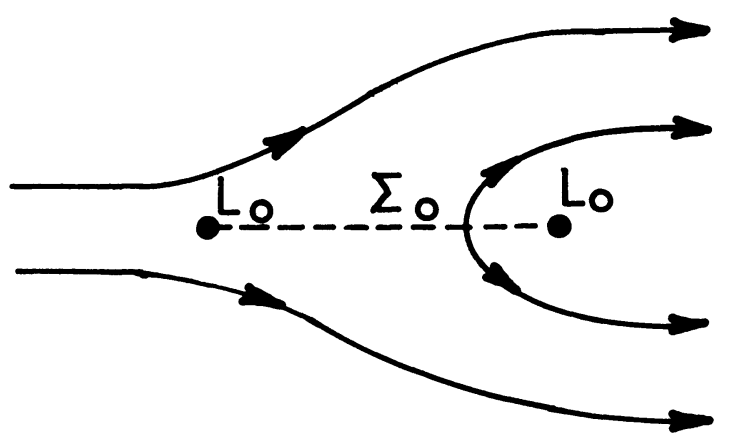

FIG. 17. - An example of mismatch surface $\Sigma_{0}$ showing the discontinuity of magnetization vectors. For this example, $\Sigma_{0}$ carries magnetic poles (of negative sign).

Of course, the discontinuity is not infinitely sharp, for various reasons :

a) If we had some direct (e. g. dipolar) interaction between grains, strong enough to compete with the local nematic alignment, they could build up something reminiscent of a Bloch wall. But, in the present paper, we are concerned with dilute colloids, for which such interactions are negligible.

b) A more important effect is translational diffusion of the grains: this implies widths of order $\sqrt{D t}$ where $D$ is a grain diffusion coefficient, and $t$ the

(5) A similar discussion, linking walls and loops of rotation disclinations, has already been carried out for solid state ferromagnets and helimagnets by M. Kleman (Phil. Mag., to be published). 
duration of the experiment. $D=10^{-8} \mathrm{~cm}^{2} / \mathrm{s}$ and $t=1 \mathrm{~s}$ would correspond to $\sqrt{D t}=1$ micron, a very sizeable thickness.

Let us assume for the moment that the surfaces of discontinuity are sharp: The situation is then similar to that of imperfect translation dislocations in ordered alloys, as has been pointed out to us by J. Friedel. (The surface of magnetic mismatch is equivalent to a surface of stacking fault for translation dislocations.) But in our case, there is essentially no "mismatch" energy associated with $\Sigma_{0}$.

Thus we expect the disclination loops to be quite mobile, just as in a conventional nematic.

When a loop $L$ moves, it sweeps a surface $\Gamma$, as shown on figure 18 , and the surface of discontinuity becomes $\Sigma_{0}+\Gamma\left({ }^{6}\right)$. Thus a moving loop leaves a « trail » behind it.
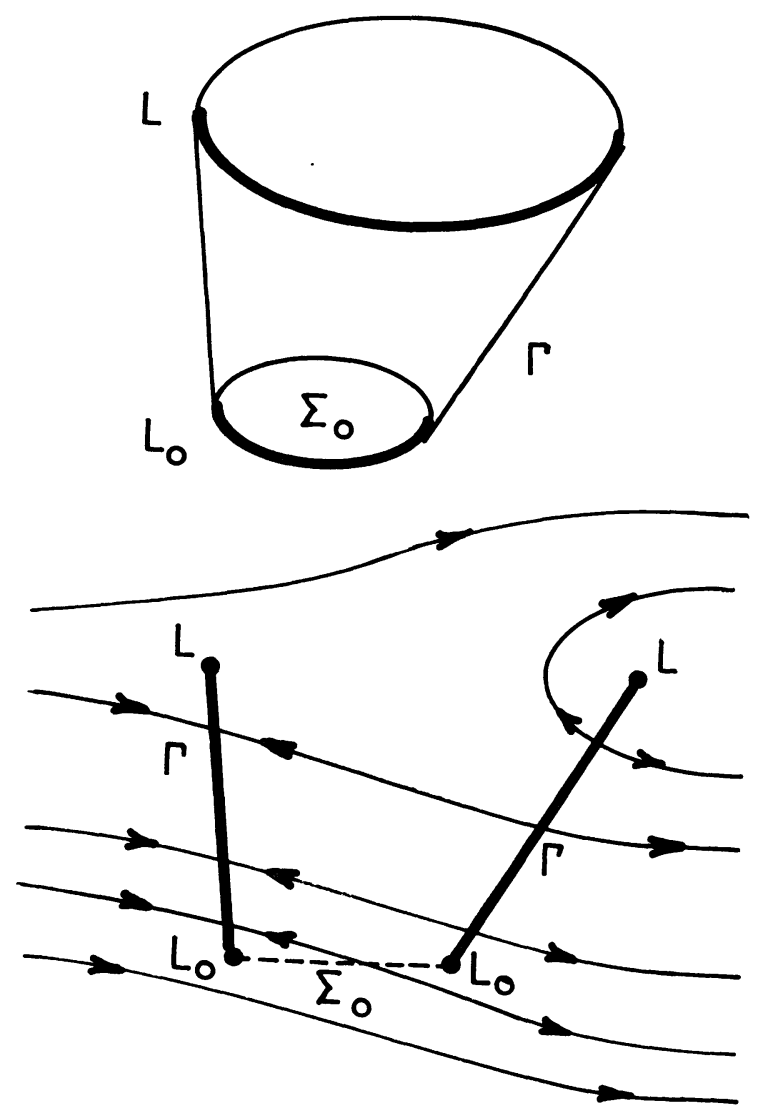

FIG. 18. - Motion of a disclination loop in a ferronematic.

It is difficult to predict what could be the nucleation and growth processes for such lines in a nematic slab under reversed fields. Let us, for simplicity, restrict our attention to cases where segregation is negligible $(\rho<1)$. As noted earlier, if $|H| \gg H_{1}$, the magnetic coherence length $\xi(H)$ is small compared to the sample thickness, and the initial conformation near one wall is that of figure $19 a$. Starting from this, one possi-

(6) Note however that, in the presence of an external field $H$ magnetic stresses may distort the surface of discontinuity. a)

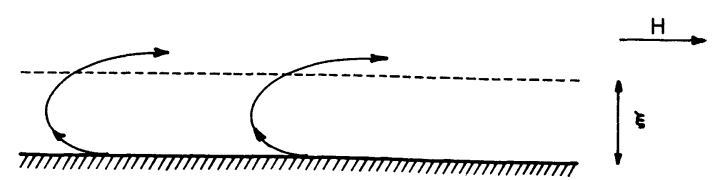

b)

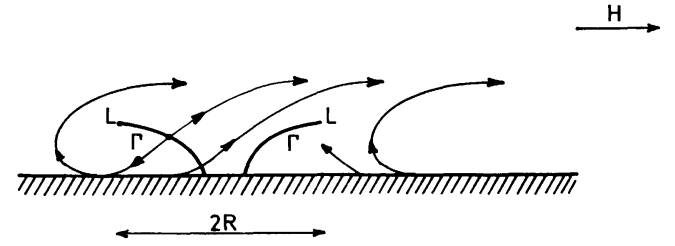

FIG. 19. - Nematic conformation in reversed fields (a) without disclination $b$ ) with a disclination loop $L$ in a plane parallel to the wall and normal to the sheet.

ble sequence of events would be the following: a loop nucleates at the surface, moves towards the inside of the sample up to a thickness of order $\xi$, and simultaneously expands in the slab plane (Fig. 19b). If all the wall surface is swept by such loops, we arrive at a situation where the nematic alignment is uniform. But the magnetization is still in the wrong direction in a sheath of thickness $\xi$ below each wall (Fig. 20). These sheaths might either smear out by translational diffusion, or show further instabilities due to the magnetic stresses.

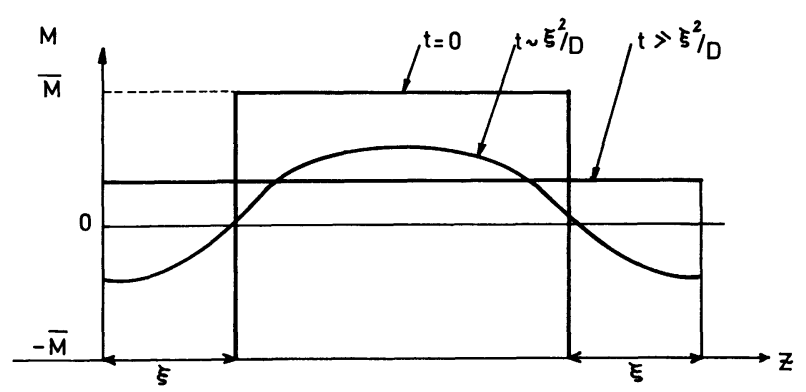

FIG. 20. - Smearing out of the magnetization profile by diffusion for a ferronematic, in reversed fields, after sweeping by disclination lines (limit $\rho<1$ )

This scheme is highly conjectural (clearly direct optical observations would be desirable). But it is worth while to observe that with such a scheme, and if the sheaths smear out by diffusion, the magnitude of the sample moment in the final state is smaller than the saturation moment, as is obvious on figure 20 . To restore the full saturation moment, one might require $|H| \sim H_{\text {mech }}$, i. e. fields large enough to rotate the individual grains.

III. 3 FerrocholeSterics. - III. 3.a Definition of the model. - We call « ferrocholesteric » a cholesteric suspension where the magnetization vector $\mathbf{M}$ spirals in phase with the local optical axis. If we call $z$ the 
spiral axis, this corresponds to the zero field configuration

$$
\begin{aligned}
M_{x} & =\bar{M} \cos q_{0} x=\bar{M} n_{x} \\
M_{y} & =\bar{M} \sin q_{0} x=\bar{M} n_{y} \\
M_{z} & =\bar{M} n_{z}=0 .
\end{aligned}
$$

The mechanical periodicity corresponds to the half pitch $\pi / q_{0}$, while the magnetic periodicity corresponds to the full pitch $2 \pi / q_{0}$. It must be emphasized that the conformation (III.27) is perfectly compatible with a strong translational brownian motion of the grains. When one grain moves around, its mechanical axis (and the moment $\boldsymbol{\mu}$ which is rigidly linked to it) follows adiabatically the local axis of molecular alignment $\mathbf{n}(\mathbf{r})$ : thus the inhomogeneous magnetization distribution (III.27) is unaffected.

It is not at all obvious, however, that the conformation (III.27) can be prepared by a simple experimental procedure : if we mixed the magnetic grains to the cholesteric in zero fields we would get at each point an equal number of grains pointing along $+\mathbf{n}$ or along $-\mathbf{n}$, and $\mathbf{M}=0$. We shall return to this preparation problem later; for the moment, we simply assume the existence of a ferrocholesteric sample, and study its response to a magnetic field $H$. We restrict our attention to the domain $H<H_{\text {mech }}$, i. e. to cooperative rotations.

The field $\mathbf{H}$ being taken in the $x$ direction, we postulate that (1) the spiral axis stays along $z$; (2) the director $\mathbf{n}$ stays in the $x y$ plane.

$$
n_{x}=\cos \varphi(z) \quad n_{y}=\sin \varphi(z) .
$$

(This excludes « conical» structures [9] : we discuss those briefly in the appendix.) With these assumptions, the free energy density has the form :

$$
\begin{aligned}
F=\frac{1}{2} K_{22}\left[\left(\frac{\mathrm{d} \varphi}{\mathrm{d} z}\right)^{2}-2 q_{0} \frac{\mathrm{d} \varphi}{\mathrm{d} z}\right] & -f M_{s} H \cos \varphi+ \\
+ & \frac{f k_{B} T}{V} \log f
\end{aligned}
$$

The equilibrium conditions are

$$
\begin{aligned}
& \psi=\frac{f}{\bar{f}}=\psi_{0} \mathrm{e}^{\rho \cos \varphi} \\
& -\xi^{2} \frac{\mathrm{d}^{2} \varphi}{\mathrm{d} z^{2}}+\psi \sin \varphi=0
\end{aligned}
$$

The constant $\psi_{0}$ is fixed by the number of grains. If we restrict our attention to periodic solutions $(\varphi(z+B) \equiv \varphi(z)+2 \pi)$ the equation defining $\psi_{0}$ may be written as

$$
\frac{1}{B} \int_{0}^{B} \psi \mathrm{d} z=1
$$

We shall now construct the solutions of (III.29, 30).
III.3.b General formulas. - Eq. (III.29) has the first integral

$$
\delta^{2}\left(\frac{\mathrm{d} \varphi}{\mathrm{d} z}\right)^{2}+\psi(\varphi)=a
$$

where $\delta$ is defined by (III.19), and is independent of $H . a$ is an (unknown) integration constant. Integrating once more we arrive at a relation between $\varphi$ and $z$ :

$$
z=\delta \int \mathrm{d} \varphi \frac{1}{\sqrt{a-\psi}}
$$

This gives us a first equation for the pitch $B$ :

$$
\frac{B}{\delta}=\int_{0}^{2 \pi} \mathrm{d} \varphi \frac{1}{\sqrt{a-\psi}}
$$

Another équation relating $B$ and $\psi_{0}$ is derived from eq. (III.30)

$$
\frac{B}{\delta}=\int_{0}^{2 \pi} \mathrm{d} \varphi \frac{\psi}{\sqrt{a-\psi}} .
$$

Comparing (III.31) and (III.33) we reach the condition

$$
\frac{B}{\delta}(a-1)=v=\int_{0}^{2 \pi} \mathrm{d} \varphi \sqrt{a-\psi} .
$$

We determine $a$ by minimization of the free energy $\mathscr{F}$ (eq. III.10) : after a slight manipulation using eq. (III.31) we arrive at

$$
\frac{\mathfrak{F}}{\Omega \bar{M} H}=\frac{\delta v}{\rho B}-\frac{2 \pi q_{0} \xi^{2}}{B}+\frac{1}{\rho} \log \psi_{0}
$$

where $\Omega$ is the sample volume.

The condition of stationary $\mathcal{F}$ leads to :

$$
\frac{\mathrm{d} a}{\rho}+\frac{2 \pi q_{0} \xi^{2} \mathrm{~d} B}{B^{2}}+\frac{1}{\rho} \frac{\mathrm{d} \psi_{0}}{\psi_{0}}=0 .
$$

We obtain $\mathrm{d} \psi_{0}$ by a differentiation of eq. (III.35)

$$
\begin{aligned}
\frac{\mathrm{d} B}{B}+\frac{\mathrm{d} a}{a-1} & =\frac{1}{2 v} \int_{0}^{2 \pi} \mathrm{d} \varphi\left[\mathrm{d} a-\psi \frac{\mathrm{d} \psi_{0}}{\psi_{0}}\right] \frac{1}{\sqrt{a-\psi}} \\
& =\frac{1}{2(a-1)}\left(\mathrm{d} a-\frac{\mathrm{d} \psi_{0}}{\psi_{0}}\right) \\
\frac{\mathrm{d} \psi_{0}}{\psi_{0}} & =-\mathrm{d} a-2(a-1) \frac{\mathrm{d} B}{B} .
\end{aligned}
$$

Inserting this result into (III.37) we arrive at the extremum condition

$$
\begin{aligned}
a-1 & =\pi q_{0} \rho \xi^{2} / B \\
& =2 \pi q_{0} \delta^{2} / B .
\end{aligned}
$$

Eq. (III.33, 34, 39) are three equations for the 
unknowns $a, B$ and $\psi_{0}$. It is often convenient to insert (III.39) into (III.35), obtaining

$$
\frac{1}{2 \pi} \int_{0}^{2 \pi} \mathrm{d} \varphi \sqrt{a-\psi}=q_{0} \delta
$$

We shall now try to get some physical insight for these results, by an investigation of various limiting cases.

III.3.c Cholesteric-Nematic transition. - In analogy with the familiar situation without grains [10] we expect the transition to be reached when $B \rightarrow \infty$. Eq. (III.35) then shows that $a=1$. To get an infinite $B$ from eq. (III.33) one then requires $\psi_{0}=\mathrm{e}^{-\rho}$ (to ensure that the integrand diverges at $\varphi=0$ ). Inserting these results into eq. (III.40) we arrive at the condition $S(\rho)=\frac{1}{2 \pi} \int_{0}^{2 \pi} \mathrm{d} \varphi \sqrt{1-\exp (\rho \cos \varphi-\rho)}=q_{0} \delta$.

The parameter $\rho$, as defined in eq. (III.9) is directly proportional to the magnetic field. Thus eq. (III.41) is an implicit equation for the critical field. A plot of $S(\rho)$ is shown in figure 21 . For $\rho \ll 1, S(\rho) \rightarrow \frac{2 \sqrt{2 \rho}}{\pi}$. For $\rho \gg 1, S(\rho)=1-C / \sqrt{\rho}$, where

$$
C=\frac{1}{2 \pi} \int_{-\infty}^{\infty} \mathrm{d} u\left[1-\sqrt{\left.1-\exp \left(-\frac{u^{2}}{2}\right)\right]} \cong 0,3\right. \text {. }
$$

We see on figure 21 that $S(\rho)$ is always smaller than unity. This means that, even with high fields, we can induce the cholesteric-nematic transition only if $q_{0} \delta<1$.

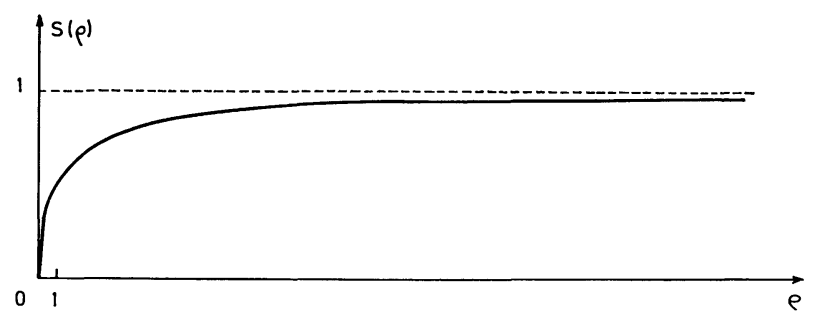

FIG. 21. - Relation between imperturbed pitch $2 \pi / q_{0}$ and critical field $H_{\mathrm{c}}=\rho k_{B} T / \mu$.

Numerically, it may be worthwhile to write down the limiting form of (III. 41) for $q_{0} \delta \ll 1$. It corresponds to a critical field

$$
H_{\mathrm{c}}=\frac{\pi^{2}}{16} \frac{K_{22} q_{0}^{2}}{\bar{M}} \quad\left(q_{0} \delta \ll 1\right) .
$$

Note that $H_{\mathrm{c}}$ is proportional to $q_{0}^{2}$ : this is strikingly different from the behaviour of conventional cholesterics (without magnetic colloids) where $H_{\mathrm{c}}$ is linear in $q_{0}$ [10]. Taking $\pi / q_{0}=80$ microns (a large value, to ensure that $q_{0} \delta \ll 1$ ) and $\bar{M}=0.1$ gauss we find from (III.42) a critical field $H_{\mathrm{c}} \sim 1$ gauss.
In the same limit $q_{0} \delta \ll 1$ we have also computed the magnetization curve (Fig. 22) and the change of pitch vs $H$; both of them can be obtained simply from eq. (III.33, 34, 40) by manipulation of elliptic integrals; the detailed calculations are described in appendix A.

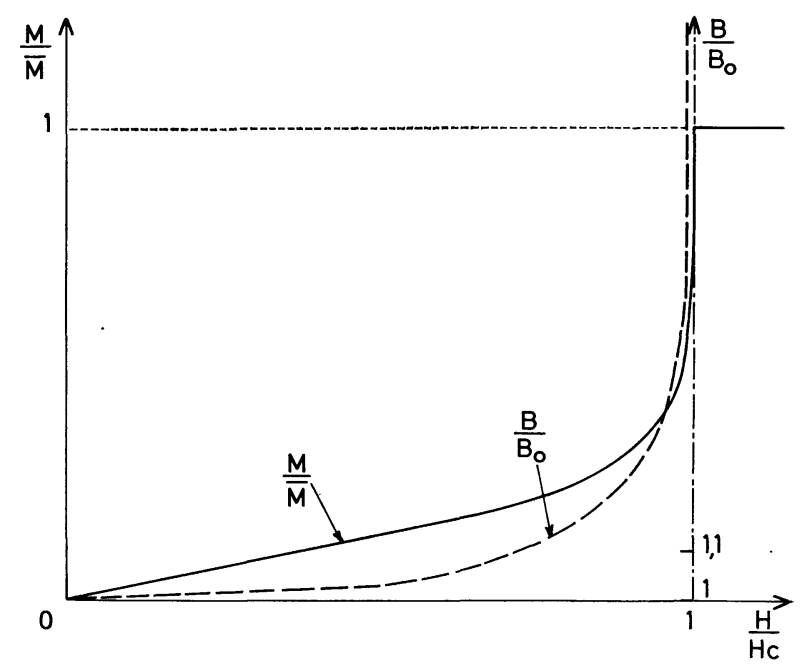

Fig. 22. - Magnetization and change of pitch vs $H$ in the limit $q_{0} \delta \ll 1$.

We shall now proceed to the opposite limit $q_{0} \delta>1$, which is somewhat less familiar.

III.3.d Layered structures. - When $q_{0} \delta \geqslant 1$ we cannot untwist completely the cholesteric structure by a field $H$. In fact, for high $H$, (high $\rho$ ) we then expect to reach a remarkable field independent conformation displayed in figure 23. This is made of successive strips, normal to the spiral axis $z$ :

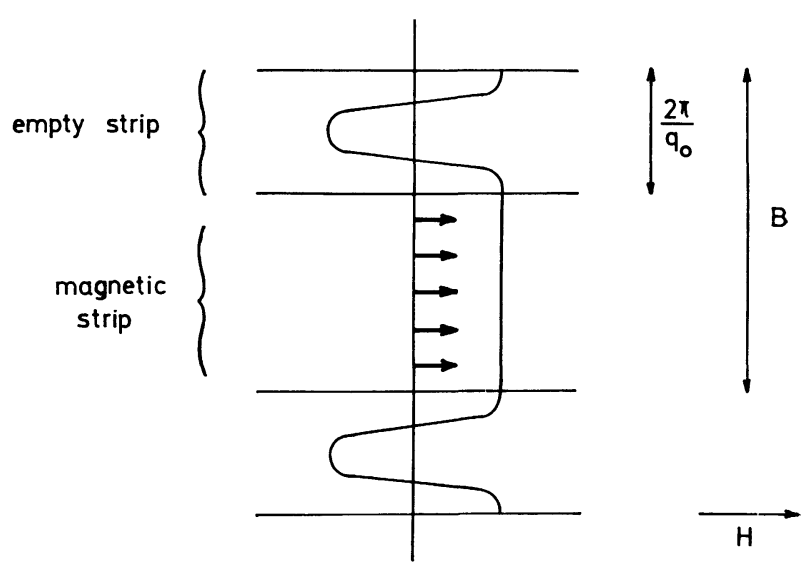

FIG. 23. - Ferrocholesteric conformation in a field $\mathrm{H}$ such that $\rho>1$ but $H<H_{\text {mech }}$ (Regime $q_{0} \delta>1$ ).

- empty strips (no magnetic grains) made of one full pitch of the unperturbed cholesteric spiral, with thickness $e=2 \pi / q_{0}$;

- magnetic strips with an increased grain concentration, all grains and molecules being aligned in the field direction. 
The average free energy (per unit volume) for such a layered system is simply

$$
\frac{\mathcal{F}^{\prime}}{\Omega}=-\frac{1}{2} K_{22} q_{0}^{2} \frac{e}{B}+c T k_{B} \log \frac{B}{B-e}+\frac{\mathscr{F}_{0}}{\Omega} .
$$

The first term in (III.43) is the gain of energy by twist in the empty strip. The second term describes the osmotic pressure effects ( $c$ is the bulk grain concentration). The last term $\mathscr{F}_{0} / \Omega$ contains the magnetic energy $-\bar{M} H$ and other contributions, all of which are independent of $B$. Minimizing $\mathbf{F}$ with respect to $B$ we find

$$
B=\frac{2 \pi q_{0} \delta^{2}}{\left(q_{0} \delta\right)^{2}-1} \quad(\rho \gg 1) .
$$

The structure can exist only for $q_{0} \delta \geqslant 1$. It can be verified that (III.44) agrees with the general formulas (III. 33, 35, 40), with the following choice of parameters :

$$
\begin{aligned}
& a=\left(q_{0} \delta\right)^{2} \\
& \psi_{0}=\mathrm{e}^{-\rho}\left(q_{0} \delta\right)^{2} \\
& \rho \gg 1 .
\end{aligned}
$$

III. 4 COMPENSATED SUSPENSIONS. - We say that a liquid crystal suspension is compensated when, at each point, in zero field, there are just as many grains with their moments pointing along $-\mathbf{n}$ or $+\mathbf{n}$ (no macroscopic magnetization for $H=0$ ). However, if, starting from such a system, we switch on a magnetic field $H$, we predict a non trivial magnetic response. This will be shown below on some specific examples.

Consider first a nematic single crystal, with all molecules aligned in the $z$ direction, and a grain fraction $\bar{f}$, corresponding to $f_{+}=\bar{f} / 2$ grains polarised along $(+z)$, and $f_{-}=\bar{f} / 2$ grains polarized along $(-z)$. We put a weak field $H$ along $z$. The unperturbed configuration becomes metastable. One configuration of lower energy (which may well turn out to be realized in practice) is displayed on figure 24 . It contains a $180^{\circ}$ wall separating the sample into two halves. There are still two families of grains (with respective distributions $f_{+}(x)$ and $f_{-}(x)$, having their magnetic moments respectively parallel or antiparallel to the local director $\mathbf{n}$. At low fields $\left(H \ll H_{\text {mech }}\right)$ a grain cannot commute from one family to the other: for instance the average of $f_{+}$on the sample volume remains fixed and equal to $f / 2$. But $f_{+}$will be large only in that half of the sample (called $A$ on Fig. 24) where $\mathbf{n}$ is parallel to $\mathbf{H}$. In the other half $(B) f_{+}$will be small. More precisely, we have

$$
\begin{aligned}
& f_{+}(\mathrm{A})=f_{-}(\mathrm{B})=\frac{\bar{f} e^{\rho}}{2 \operatorname{ch} \rho} \\
& f_{-}(\mathrm{A})=f_{+}(\mathrm{B})=\frac{\bar{f} e^{-\rho}}{2 \operatorname{ch} \rho} .
\end{aligned}
$$

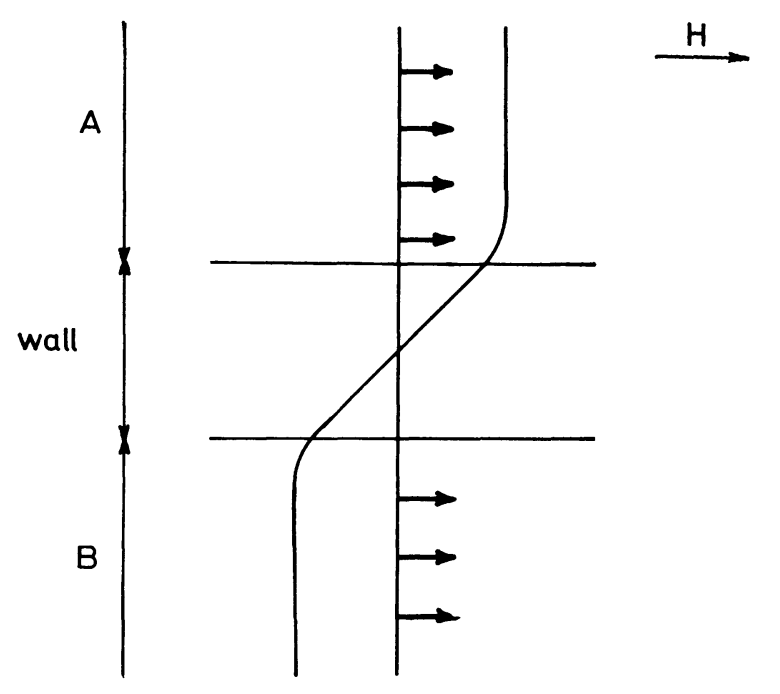

FIG. 24. - Compensated nematic under a field : separation in two phases.

In particular, for high $\rho$, the new configuration corresponds to complete demixtion of the two types of grains.

The macroscopic free energy $\mathscr{F}_{\mathrm{d}}$, associated with the distribution (III.45) is given by

$$
\begin{aligned}
& \frac{\mathscr{F}_{\mathrm{d}}}{\Omega}=\frac{k_{B} T}{2 V}\left[f_{+}(\mathrm{A}) \log f_{+}(\mathrm{A})+f_{-}(\mathrm{A}) \log f_{-}(\mathrm{A})+\right. \\
& \left.+f_{+}(\mathrm{B}) \log f_{+}(\mathrm{B})+f_{-}(\mathrm{B}) \log f_{-}(\mathrm{B})\right]- \\
& -M_{s} H\left[f_{+}(\mathrm{A})-f_{-}(\mathrm{A})-f_{+}(\mathrm{B})+f_{-}(\mathrm{B})\right]= \\
& =\frac{k_{B} T \bar{f}}{V} \log \left(\frac{\bar{f}}{2 \operatorname{ch} \rho}\right) \text {. }
\end{aligned}
$$

For comparison the free energy of the initial phase was simply

$$
\mathscr{F}_{0}=\frac{\Omega k_{B} T \bar{f}}{V} \log \frac{\bar{f}}{2}=N k_{B} T \log \frac{\bar{f}}{2}
$$

where $N$ is the total number of grains. Thus

$$
\mathscr{F}_{\mathrm{d}}-\mathscr{F}_{0}=-N k_{B} T \log [\mathrm{ch} \rho]<0 \text { (III.48) }
$$

and the initial phase is indeed metastable $\left({ }^{7}\right)$.

Of course, in practice, a number of physical problems would arise, which are not solved by the above elementary considerations :

(1) other conformations may be more favourable energetically than our " one wall structure" ;

(2) even if the one wall structure is indeed lowest in free energy, the nucleation of the wall may require comparatively high $H$. But in any case our result shows that even compensated solutions may show remarkable effects in the presence of a magnetic field.

(7) The structure of the wall separating regions $A$ and $B$ can be derived simply from a generalization of eq. (III.11) to two-component grain systems. When $\rho>1$ the thickness of the wall is proportional to $\delta$. 
This is also true for compensated cholesteric suspensions. Consider for instance the regime $q_{0} \delta>1$ and $\rho>1$ for such a system. Here, instead of having the layered structure of figure 23 we expect a splitlayer conformation (Fig. 25) with two $180^{\circ}$ walls (containing no grains) and two nematic bands (containing respectively the + and the - families) per unit cell. The overall period $B$, however, is unchanged, this can be understood as follows: the difference in free energy between figure 25 and figure 23 is only due to the entropy of mixing for the + and - families, and is $N k_{B} T \log 2$ (independent of $B$ ). Thus minimization with respect to $B$ will again lead to eq. (III.44).
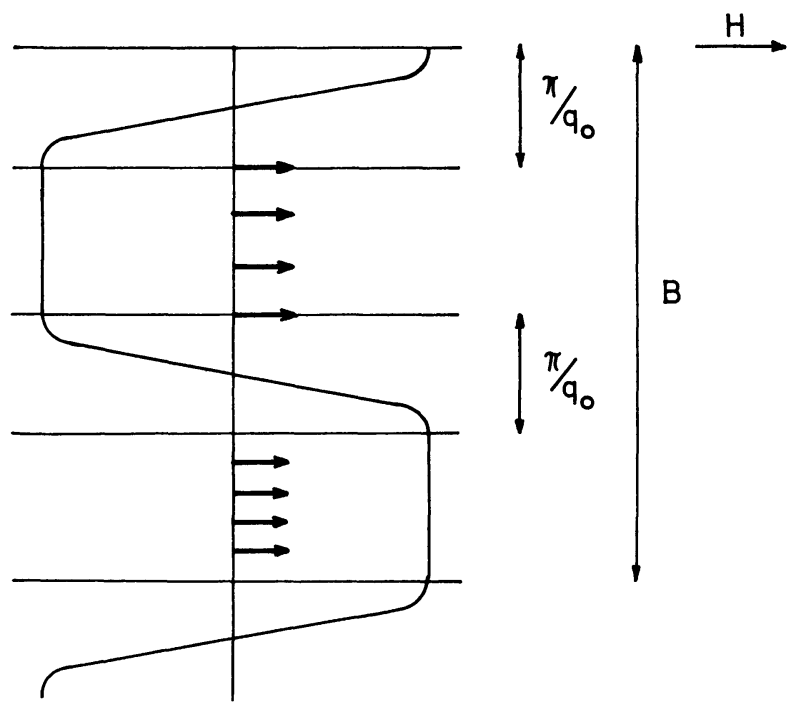

FIG. 25. — «Split layer» conformation for a compensated cholesteric.

IV. Discussion. - Magnetic colloids could be an efficient mean of coupling a liquid crystal to a weak magnetic field. There are, however, certain difficulties associated with this method, which we list below.

1) INCORPORATION OF THE GRAINS in the (non-polar) liquid crystal : our hope is that this can be achieved with small grains coated by a suitable detergent [11]. However, with the flexible detergent molecules covering the surface, it is not obvious the direction of $\mathbf{n}$ is rigidly fixed at each point of this surface. If it is not rigidly fixed, the distortion amplitude $l(\theta)$ will become much smaller than the grain length $L$ : the coupling between grain axis and nematic axis is then strongly decreased with respect to our estimates of section II $\left({ }^{8}\right)$.

2) OPTICAL OBSERVATION OF THE CONFORMATIONS. This would be one of the best tools of investigation. But, if (as usual) the grains are essentially opaque,

(8) A similar difficulty might occur if there are too many disclination loops tied to each grain. However, this can probably be avoided by cooling the samples from the isotropic phase, under a strong field (see 4, below). such observations require very thin samples: the optical free path $\Lambda$ is of order $1 / c \sigma$ where $c=f / L d^{2}$ is the grain concentration and $\sigma=L d$ is the lateral surface of one grain. Thus $\Lambda \sim d / f$ is of order 20 microns for $f=10^{-3}$ and $d=200 \AA$.

3) EFFECTS of INTERACTIONS BETWEEN GRAINS. a) When the grains are too large, it is impossible to make a stable colloid. Even below the critical size, they tend to line up in chains.

In some approximate sense, the chains will behave as larger grains : they will still provide a strong coupling between $\mathbf{H}$ and $\mathbf{n}$. But the effective segregation parameter $\rho$ will be very large (increasing like the number of grains in one chain).

b) On a different, more macroscopic, scale, we have constantly ignored the effects of the dipolar interactions between grains; this will be valid only when all external fields of interest are larger than the demagnetizing fields $(H>\bar{M})$.

4) PRePaRATION OF « FERRO» LIQUIDS AND « COMPENSATED » LIQUIDS. - One of the major conclusions of section III is the following : the properties of a magnetic suspension in a liquid crystal are extremely sensitive to the previous history of the sample. Thus one must always use a careful preparation procedure to obtain a meaningful result :

a) To generate a ferronematic one should use a specimen oriented by the method of Chatelain [6] between two polished plates, and start with a field $H>H_{\text {mech }}$ applied along the easy axis. It may also help to cool the isotropic phase down to nematic temperatures under those conditions to avoid disclination loops in the local arrangement near each grain.

b) What do we get if we start from $H>H_{\text {mech }}$ with a cholesteric substrate ? Let us discuss first the case of large unperturbed pitches $\left(q_{0} \delta<1\right)$. Then the high field state is nematic. When $H$ is decreased we may find either one of the following two transitions

$$
\begin{aligned}
& (\alpha) \quad \text { nematic } \rightarrow \text { conical structure } \\
& (\beta) \quad \text { nematic } \rightarrow \text { cholesteric }
\end{aligned}
$$

The numerical conditions required to find $(\alpha)$ or $(\beta)$ are discussed in appendix B. In case $(\alpha)$ the final conformation is derived continuously from the high field ferronematic : thus it is ferrocholesteric. In case $(\beta)$, depending on the detailed conditions, the cholesteric phase is known to nucleate either through disclination loops [12] or through the growth of a wall which had remained as a metastable object in the high field phase [9]. The latter process is depicted on figure 26. Disclinations of rank 1 should be avoided: as seen in section III a loop $L$ generates a surface of mismatch $\Sigma$ around which diffusion processes cancel out the magnetization. On the other hand, the wall process involves no discontinuity and appears to be acceptable. 


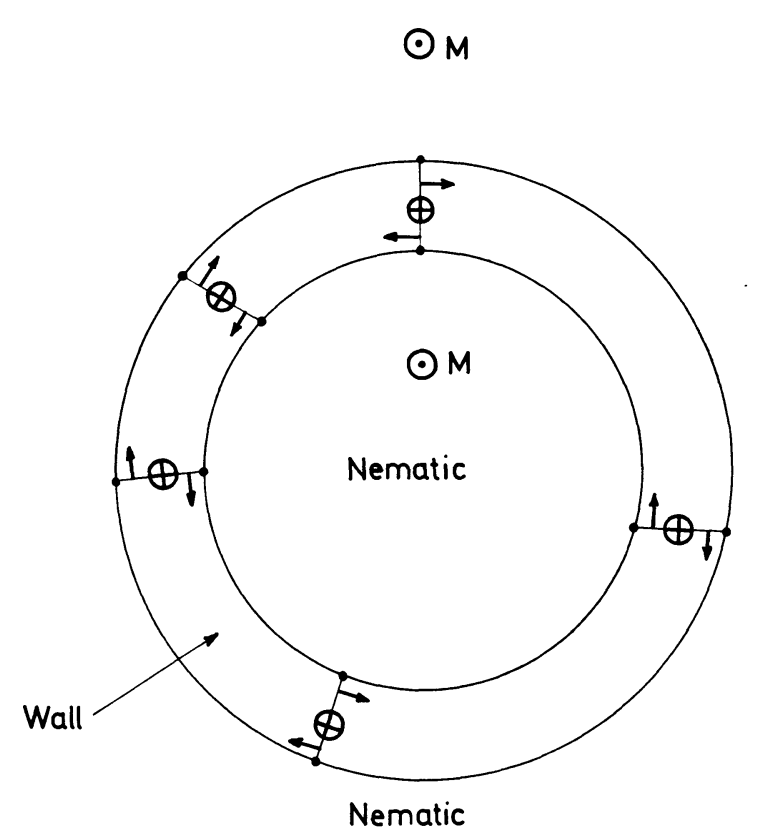

FIG. 26. - The Meyer type of nucleation center for the nematic cholesteric transition (after ref. 9. transposed here to the case of a ferrocholesteric). Above $H_{\mathrm{c}}$ the cylindrical wall is shrunk but cannot disappear. Below $\boldsymbol{H}_{\mathrm{c}}$ it increases its surface indefinitely and fills all the sample volume.

c) If we start from $H>H_{\text {mech }}$ in a cholesteric of small pitch $\left(q_{0} \delta>1\right)$ the initial conformation corresponds to figure 25 with nematic layers separated by (empty) $180^{\circ}$ walls. When $H$ is decreased this will give rise to a compensated cholesteric. To prepare a ferrocholesteric of small pitch more sophisticated methods should be used. One possibility is to have the pure cholesteric $C$ in contact with an isotropic liquid $I$, the grains being initially in $I$ (and polarised by a weak field $H$ ). If $C$ is a better solvent than $I$ for the grains, they will diffuse in $C$ and one could reach a ferrocholesteric state.

5) Contamination efFects. - If a nominal ferronematic is really only partially saturated (i. e., at each point, $f_{+} \gg f_{-}$but $f_{-} \neq 0$ ) some of our conclusions in section III-2 become totally invalid. For twisted configurations near a wall (and $\rho>1$ ) we predicted a depletion layer of thickness $\delta\left(f_{+} \rightarrow 0\right.$ in the layer). However, it must be realized that this layer acts as a trap for the minority carriers $\left(f_{-}\right)$. Thus, even if there are but few minority carriers in the bulk, they may saturate the layer and change completely its optical properties.

To conclude : the physics of magnetic suspensions in liquid crystals will require a long and careful experimentation involving both physicists and physicalchemists. But these experiments should display a number of rather remarkable phase transitions.

Apart from magnetic grains, there are also many other " floating objects " which could give interesting effects in liquid crystals. One example is polypeptide rods, carrying a strong electric dipole moment : dilute solutions of such rods might be sensitive to low electric fields. We hope to come back to some of these problems in later work.

Acknowledgments. - We have greatly benefited from discussions with J. P. Burger, P. Cladis, G. Durand, J. Friedel, F. Hartmann, M. Kléman, J. Rault, M. Veyssié.

Appendix A. - Distortion of a ferrocholesteric structure in the limit $q_{0} \delta \ll 1$.

The equilibrium condition (III.29), in the limit $\rho \ll 1$, may be written

$$
-\xi^{2} \frac{\mathrm{d}^{2} \varphi}{\mathrm{d} z^{2}}+\sin \varphi=0 .
$$

This equation has for first integral

$$
\xi^{2}\left(\frac{d \alpha}{d z}\right)+\sin ^{2} \alpha=\frac{1}{k^{2}}, \quad \text { where } \quad \alpha=\frac{\varphi}{2}-\frac{\pi}{2} .
$$

The pitch $B$ of the structure corresponds to a variation $\Delta \varphi=2 \pi$ :

$$
B=\int_{0}^{2 \pi} \mathrm{d} \varphi \frac{\mathrm{d} z}{\mathrm{~d} \varphi}=k \xi \int_{-\pi / 2}^{\pi / 2} \frac{\mathrm{d} \alpha}{\sqrt{1-k^{2} \sin ^{2} \alpha}} .
$$

Eq. (III.40), the condition of stationary $f$ with respect to $1 / k^{2}$ leads to :

$$
\pi q_{0} \xi=2 \int_{-\pi / 2}^{\pi / 2} \frac{\mathrm{d} \alpha}{k} \sqrt{1-k^{2} \sin ^{2} \alpha}=4 \frac{E(k)}{k},
$$

using elliptic integral formulation [7].

Eq. (I) and (II) give the relationship between $B$ and $H$ (Fig. 22). As the field's strength approaches a critical value

$$
H_{\mathrm{c}}=\frac{\pi^{2}}{16} \frac{K_{22} q_{0}^{2}}{\bar{M}}
$$

the pitch diverges logarithmically. For $H>H_{\mathrm{c}}$, the structure is converted to a nematic one. The pitch is much more sensitive to the field than for a pure liquid crystal. For small values of $H$, the variation of $B$ is proportional to $H^{2}$

$$
\frac{B}{B_{0}}=1+\frac{\pi^{2}}{8} \frac{H^{2}}{H_{c}^{2}}
$$

instead of $H^{4}$.

The magnetization per $\mathrm{cm}^{3}$ in the field direction is given by

$$
\frac{M}{\bar{M}}=\frac{\int_{0}^{2 \pi}-\cos \varphi \frac{\mathrm{d} z}{\mathrm{~d} \varphi} \mathrm{d} \varphi}{\int_{0}^{2 \pi} \frac{\mathrm{d} z}{\mathrm{~d} \varphi} \mathrm{d} \varphi}=\frac{2}{k^{2}}\left|1-\frac{E(k)}{K(k)}\right|-1 .
$$


Eq. (II) and (III) give the relationship between $M / \bar{M}$ and $H / H_{\mathrm{c}}$ (Fig. 22). $M$ is a linear function of $H$ for small fields

$$
\frac{M}{\bar{M}}=\frac{\pi^{2}}{32} \frac{H}{H_{\mathrm{c}}},
$$

As $H \rightarrow H_{\mathrm{c}}, M / \bar{M} \rightarrow 1$ with a vertical tangent.

The continuity of $M=f(H)$ proves that the transition is second order.

Appendix B. - Conical structures in ferrocholesterics.

In section III. 3 we have restricted our attention to spirals with the field $\mathbf{H}$ normal to the spiral axis $z$. We now discuss briefly the opposite case, where $\mathbf{H}$ is parallel to $z$ and look for solutions of the form

$$
\left.\begin{array}{l}
n_{x}=\sin \theta \cos q z \\
n_{y}=\sin \theta \sin q z \\
n_{z}=\cos \theta=\text { constant } .
\end{array}\right\}
$$

For such a configuration there is no segregation since the energy of one grain $(-\mu H \cos \theta)$ is independent of its location. The free energy density is

$F=\sin ^{2} \theta\left[\frac{1}{2} K(\theta) q^{2}-K_{22} q q_{0}\right]-\bar{M} H \cos \theta+$ const. where $K(\theta)=K_{22} \sin ^{2} \theta+K_{33} \cos ^{2} \theta$. Minimizing $F$ with respect to $q$ one arrives at

$$
F=-\frac{\left(K_{22} q_{0} \sin \theta\right)^{2}}{2 K(\theta)}-\bar{M} H \cos \theta .
$$

Let us start from the nematic phase in high fields (assuming $q_{0} \delta<1$ ) and investigate the stability with respect to a small conical distortion $(\theta \rightarrow 0)$.

We have :

$$
\begin{aligned}
F-F_{\text {nematic }} & =\frac{1}{2} \theta^{2}\left[-\frac{\left(K_{22} q_{0}\right)^{2}}{K_{33}}+\bar{M} H\right] \\
& =\frac{1}{2} \theta^{2{ }^{7}} K_{22} q_{0}^{2}\left[-\frac{K_{22}}{K_{33}}+\frac{\rho}{2 \delta^{2} q_{0}^{2}}\right]
\end{aligned}
$$

conical structures will not appear if the coefficient of $\theta^{2}$ stays positive for all $H \geqslant H_{\mathrm{c}}\left(H_{\mathrm{c}}=\right.$ critical field defined by eq. (III.41)). This condition may be written as

$$
\frac{K_{22}}{K_{33}} \leqslant G\left(q_{0} \delta\right) \rightarrow \text { no conical structures }
$$

where $G=\left[\rho / 2 S^{2}(\rho)\right]_{H=H_{\mathrm{c}}}$. The function $G$ is plotted on figure 27 .

$$
\text { For } \quad q_{0} \delta \ll 1 \quad G \rightarrow \frac{\pi^{2}}{16}
$$

For $q_{0} \delta \rightarrow 1, G$ tends to infinity.

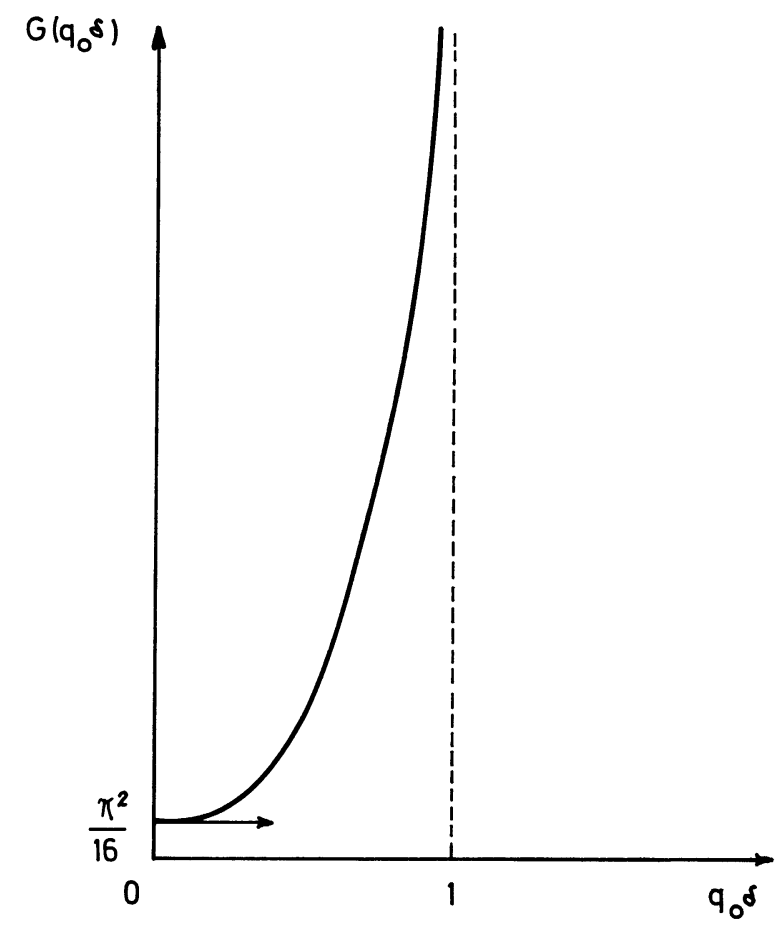

FIG. 27. - Conical structures in ferrocholesteric will appear if $K_{22} / K_{33} \geqslant G\left(q_{0} \delta\right)$.

\section{References}

[1] See for instance, Chistiakov (G.), Uspekhi Fiz. Nauk., 1966, 89, 563.

De Gennes (P. G.), Molecular Crystals, 1969, 7, 325.

[2] Frank (F. C.), Dis. Faraday Soc., 1958, 25, 1.

[3] Electrostatique et Magnétostatique, Durand, Masson editor.

[4] Cano (R.), Bull. Soc. Franç. Mineral. Cristallogr., 1967, 90, 333.

[5] Néel (L.), C. R. Acad. Sci., 1949, 228, 664 ; Ann. Géophys., 1949, 5, 99.

[6] Chatelain (P.), Bull. Soc. Franç. Mineral. Cristallogr., 1963, 66, 105.
[7] Elliptic integrals. See JAHNKe, EMde, Lösch, Tables of higher functions, B. G. Teubner Verlagsgesellschaft, Stuttgart.

[8] For a review on disclination lines, see FrIEDel (J.) Kleman (M.), J. Physique, 30, Colloque C4, Supplément au No 11-12.

[9] For a discussion of conical structures in conventional cholesterics under fields, see R. B. MEYER, Thesis, Harvard, 1969.

[10] De Gennes (P. G.), Solid State Communications, 1968, $6,163$.

[11] Cladis (P.), Rault (J.), private communications.

[12] Groupe (" Cristaux Liquides ), Orsay, Physics Letters, 1963, 28 A, 10. 